Philosophical Magazine B, 2001, Vol. 81, No. 12, 2021-2054

\title{
Domain patterns and macroscopic behaviour of ferroelectric materials
}

\author{
Y. C. SHU \\ Institute of Applied Mechanics, National Taiwan University, Taipei, Taiwan, \\ ROC
}

and K. BHATTACHARYA $\dagger$

Division of Engineering and Applied Science, 104-44, California Institute of Technology, Pasadena, California 91125, USA

[Received 18 August 2000 and accepted 28 May 2001]

\begin{abstract}
This paper examines the domain patterns and the macroscopic behaviour of single crystals of ferroelectric material using a theory based on energy minimization. A low-energy path is identified for domain switching and a novel configuration that yields very large electrostriction is identified.
\end{abstract}

\section{$\S 1$ INTRODUCTION}

Ferroelectric crystals are widely used in a variety of applications including transducers, capacitors and non-volatile data storage elements. In particular, poled ceramics of ferroelectric materials are used commonly for their piezoelectric property in actuator applications (Huber et al. 1997, Uchino 1998). Their many advantages include a linear response of the induced strain to applied electric field, very little hysteresis and very-high frequency response. The strains and displacements that they display, however, are quite small. An improvement in the strain level would lead to an explosion of the applications of these materials as miniature solid-state actuators and sensors in a variety of structural applications. It has recently been recognized that it is possible to obtain large strains through electrostriction in single crystals (Shrout and Park 1997).

Ferroelectric crystals are non-polar above the Curie temperature but are spontaneously polarized with a spontaneous lattice distortion below the Curie temperature. The interesting properties of ferroelectrics arise from the fact that it is possible to change the polarization and distortion through applied electric field or mechanical stress. Further, there is typically a reduction in crystallographic symmetry at the Curie temperature and this creates more than one symmetry-related, spontaneously polarized and distorted state below this temperature. The different states can coexist as 'domains' in very intricate and characteristic domain patterns (for example Hooton and Merz (1955), Little (1955), Tanaka and Honjo (1964), Arlt and Sasko (1980), Goo et al. (1981), Hu et al. (1986), Li et al. (1992), Park and Chung (1994,

\footnotetext{
$\dagger$ †uthor for correspondence. E-mail: bhatta@caltech.edu

Philosophical Magazine B ISSN 1364-2812 print/ISSN 1463-6417 online (C) 2001 Taylor \& Francis Ltd 
1996) and Chou and Wayman (1997)). These domains can be switched and the domain patterns manipulated by the application of electric field and mechanical stress. Domain switching with its large change in polarization and lattice distortion provides a potent mechanism for obtaining large and unusual properties.

This paper studies the domain patterns and the macroscopic properties of single crystals of ferroelectrics and proposes a new way of obtaining large electrostriction. We propose, in $\S 2$, a variational theory that describes the behaviour of a ferroelectric single crystal. We write the total energy of the crystal as a sum of the free (or stored) energy, the energy of the applied electrical and mechanical loads, the energy of the induced electric field and the domain wall energy; the state of the ferroelectric crystal is obtained as minimizers of this total energy. The stored energy density encodes phenomenologically the information that the crystal prefers a few given spontaneously polarized and distorted states and can be non-convex with multiple wells owing to the presence of multiple such states. This theory is similar to the classical Landau-Ginzburg-Devonshire theory (Devonshire 1949, 1951, 1954) (see also Fatuzzo and Merz (1967) and Lines and Glass (1979) for lucid descriptions of the theory and its successes and Damjanovic (1998) for a recent review) with a few key differences. First, we allow for finite deformations which are quite important as the strains become large. Second, we carefully account for the energy of the electric field, both applied and induced. This turns out to be quite important in deciding between differently shaped specimens. Finally, we do not restrict ourselves to polynomials but use very general functions and impose only the conditions required by symmetry.

We show in $\S 3$ that energy minimization based on this theory automatically leads to domain patterns and accurately predicts the observed domain patterns in common ferroelectric materials based on very few parameters. Energy minimization based on an energy with multiple wells leads to frustration which manifests itself as domains. The positions of the wells, the electrostatic energy and the mechanical compatibility determine very characteristic domain patterns. An important result is that many commonly observed domain walls have low energy and that this is a surprising and non-generic consequence of material symmetry. It follows from this observation that these domain walls provide a low-energy path for domain switching. This result is consistent with recent experiment ( $\mathrm{Li}$ et al. 1991, 1992) on barium titanate $\left(\mathrm{BaTiO}_{3}\right)$ single crystals which demonstrated that the critical compressive stress for the nucleation and removal of domains is below or around only $1 \mathrm{MPa}$. This section also provides a detailed analysis of many complicated domain patterns in a variety of materials and compares them with experimental observations.

This theoretical framework and analysis are similar to those used recently with great success in the study of martensitic materials (Ball and James 1987, 1992, Bhattacharya 1991, 1992, Hane and Shield 1998), magnetostrictive materials (James and Kinderlehrer 1993), and ferromagnetic shape-memory alloys (James and Wuttig 1998, DeSimone and James 1997, 2001).

In $\S 4$ we turn to the study of electrostriction through domain switching and propose a novel large strain actuator using a carefully chosen configuration and electromechanical loading path. The basic idea is that domain switching can give rise to large strains, and this can take place because of a suitable geometry and electromechanical loading path. The predictions of this section have been recently verified experimentally by Burcsu et al. (2000) for $\mathrm{BaTiO}_{3}$ single crystals. 


\section{§2. Continuum Model}

\subsection{Kinematics}

Consider a ferroelectric single crystal and an arrangement of conductors shown in figure 1. The ferroelectric crystal occupies the region $\Omega \subset \mathbb{R}^{3}$ in its reference configuration. The deformation of the crystal is described by the function $\mathbf{y}: \Omega \rightarrow \mathbb{R}^{3}$, the deformation gradient is $\mathbf{F}=\nabla_{\mathbf{x}} \mathbf{y}: \Omega \rightarrow \mathbb{M}^{3 \times 3}$ where $\mathbb{M}^{m \times n}$ is the set of all $m \times n$ matrices and $\nabla_{\mathbf{x}}$ is the gradient with respect to the reference configuration, and $\mathbf{y}(\Omega)$ is the region occupied by the crystal in the current configuration. We assume that the deformation is invertible, and that $\operatorname{det} \nabla_{\mathbf{x}} \mathbf{y}(\mathbf{x})$ exists and is positive almost everywhere in $\Omega$. Although we use a continuum theory, we have the following atomic picture in mind. Associated with the material point $\mathbf{x}$, there is a lattice with lattice vectors $\left\{\mathbf{I}_{1}^{0}, \mathbf{I}_{2}^{0}, \mathbf{I}_{3}^{0}\right\}$ in the reference configuration. As the crystal deforms, so does the lattice: therefore, after deformation, the lattice has lattice vectors $\left\{\mathbf{I}_{1}, \mathbf{I}_{2}, \mathbf{I}_{3}\right\}$. We invoke the Cauchy-Born rule (Ericksen 1984) which says that the overall distortion of the lattice follows the macroscopic distortion, or

$$
\mathbf{I}_{i}=\left(\nabla_{\mathbf{x}} \mathbf{y}\right) \mathbf{l}_{i}^{0} \quad i=1,2,3 .
$$

We find it convenient to choose the undistorted non-polar phase at the Curie temperature as our reference configuration; therefore, $\left\{\mathbf{I}_{i}^{0}\right\}$ is independent of $\mathbf{x}$.

We denote the spontaneous polarization at the point $\mathbf{y}$ in the current configuration as $\mathbf{p}(\mathbf{y})$. Since our deformation is invertible, we shall find it convenient at times to 'pull back' $\mathbf{p}$ to the reference configuration, and we write $\mathbf{p}(\mathbf{x})=\mathbf{p}(\mathbf{y}(\mathbf{x}))$ with a slight abuse of notation.

We apply a dead load to our crystal with fixed reference traction $\mathbf{t}_{0}: \partial \Omega \rightarrow \mathbb{R}^{3}$ and use an arrangement of conductors $C_{1} \subset \mathbb{R}^{3}$ with total charge $Q$ and $C_{2} \subset \mathbb{R}^{3}$ with a fixed potential $\hat{\phi}$ to apply an electric field. Let $\mathbf{e}_{0}: \mathbb{R}^{3} \rightarrow \mathbb{R}^{3}$ be the electric field that would be present owing to the conductors $C_{1}$ and $C_{2}$ were the ferroelectric crystal absent.

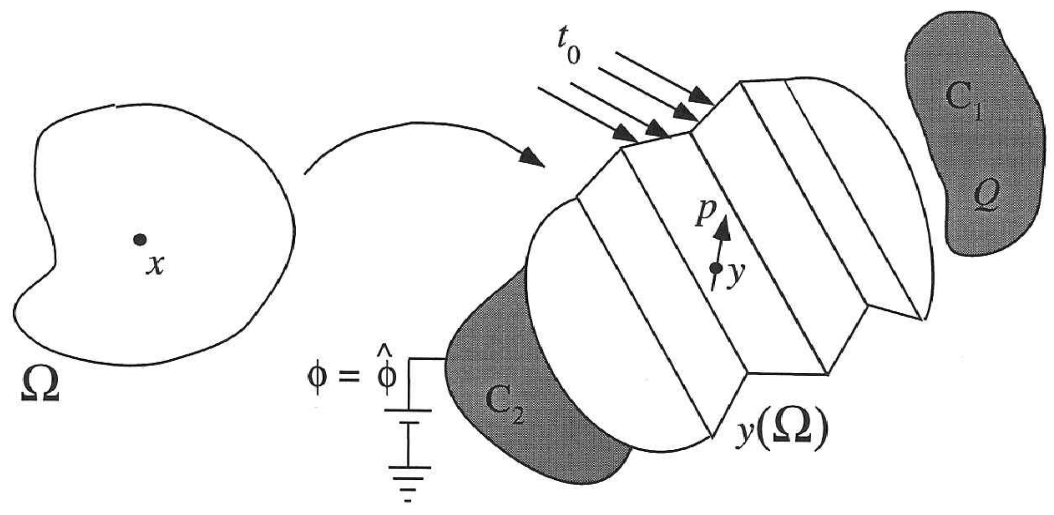

Figure 1. A ferroelectric-conductor system where $\Omega$ is the undistorted reference configuration of the ferroelectric crystal at its Curie temperature $\theta_{\mathrm{c}} ; C_{1}$ and $C_{2}$ are the domains occupied by conductors with total charge $Q$ and fixed potential $\hat{\phi}$ respectively. The crystal is subjected to a dead load $\mathbf{t}_{0}$ on some parts of its boundary. 


\subsection{Electroelastic Energy}

For a ferroelectric crystal at temperature $\theta$ subject to a dead load $\mathbf{t}_{0}$ and an electric field $\mathbf{e}_{0}$ as described above, we postulate that we can obtain the deformation and polarization as those that minimize

$$
\begin{aligned}
\mathcal{E}(\mathbf{y}, \mathbf{p})= & \int_{\Omega}\left[\frac{1}{2} \nabla_{\mathbf{x}} \mathbf{p} \cdot \mathbf{A} \nabla_{\mathbf{x}} \mathbf{p}+W\left(\nabla_{\mathbf{x}} \mathbf{y}, \mathbf{p}, \theta\right)-\mathbf{e}_{0} \cdot \mathbf{p}\left(\operatorname{det} \nabla_{\mathbf{x}} \mathbf{y}\right)\right] \mathrm{d} \mathbf{x} \\
& -\int_{\partial \Omega} \mathbf{t}_{0} \cdot \mathbf{y} \mathrm{d} S_{\mathbf{x}}+\frac{\epsilon_{0}}{2} \int_{\mathbb{R}^{3}}\left|\nabla_{\mathbf{y}} \phi\right|^{2} \mathrm{~d} \mathbf{y},
\end{aligned}
$$

where $\mathbf{A} \in \mathbb{M}^{3 \times 3}$ is a positive definite matrix, $\nabla_{\mathbf{y}}$ is the gradient with respect to the current configuration, $W: \mathbb{M}^{3 \times 3} \times \mathbb{R}^{3} \times \mathbb{R} \rightarrow \mathbb{R}$ is the given 'stored energy density', $\epsilon_{0}=8.85 \times 10^{-12} \mathrm{C}^{2} \mathrm{~N}^{-1} \mathrm{~m}^{-2}$ is the coefficient of permittivity of the free space and the electric potential $\phi$ is obtained by solving Maxwell's equation

$$
\nabla_{\mathbf{y}} \cdot\left(-\epsilon_{0} \nabla_{\mathbf{y}} \phi+\mathbf{p} \chi_{\mathbf{y}(\Omega)}\right)=\rho_{\mathrm{f}} \quad \text { on } \mathbb{R}^{3},
$$

subject to

$$
\begin{gathered}
\nabla_{\mathbf{y}} \phi=\mathbf{0} \quad \text { on } C_{1}, \quad \int_{\partial C_{1}} \frac{\partial \phi}{\partial n} \mathrm{~d} S=0, \quad \phi=0 \quad \text { on } C_{2}, \\
\phi \rightarrow 0 \quad \text { as }|\mathbf{y}| \rightarrow \infty
\end{gathered}
$$

for some free charge $\rho_{\mathrm{f}}$ supported on $\partial C_{1} \cup \partial C_{2}$.

Let us clarify the notation. Given $\mathbf{y}: \Omega \rightarrow \mathbb{R}^{3}$ and $\mathbf{p}: \mathbf{y}(\Omega) \rightarrow \mathbb{R}^{3}$, we solve equation (2) to obtain $\phi$ and substitute it back into equation (1) to obtain $\mathcal{E}(\mathbf{y}, \mathbf{p})$. Maxwell's equation (2) is of course solved over all space $\mathbb{R}^{3}$ with $\mathbf{p}=\mathbf{0}$ outside $\mathbf{y}(\Omega)$; we emphasize this by writing $\mathbf{p} \chi_{\mathbf{y}(\Omega)}$, where $\chi_{\mathbf{y}(\Omega)}$ is the characteristic function of $\mathbf{y}(\Omega)$ so that $\chi_{\mathbf{y}(\Omega)}=1$ on $\mathbf{y}(\Omega)$ and $\chi_{\mathbf{y}(\Omega)}=0$ outside $\mathbf{y}(\Omega)$. The free charge $\rho_{\mathrm{f}}$ is a measure supported on $\partial C_{1} \cup \partial C_{2}$, and we assume that it is of the form $\mathrm{d} \rho_{\mathrm{f}}=\rho_{\mathrm{f}} \mathrm{d} S$ where $\rho_{\mathrm{f}}: \partial C_{1} \cup \partial C_{2} \rightarrow \mathbb{R}$ with an obvious abuse of notation. Thus, by equation (2), we mean

$$
\begin{gathered}
\nabla_{\mathbf{y}} \cdot\left(-\epsilon_{0} \nabla_{\mathbf{y}} \phi+\mathbf{p}\right)=0 \quad \text { in } \mathbf{y}(\Omega), \\
\nabla_{\mathbf{y}}^{2} \phi=0 \quad \text { in } C_{1} \cup C_{2} \cup\left(\mathbb{R}^{3} \backslash\left(\overline{\mathbf{y}(\Omega)} \cup \overline{C_{1}} \cup \overline{C_{2}}\right)\right), \\
\llbracket-\epsilon_{0} \nabla_{\mathbf{y}} \phi+\mathbf{p} \rrbracket \cdot \mathbf{n}^{\prime}=0 \quad \text { on } \partial \mathbf{y}(\Omega) \backslash\left(\partial C_{1} \cup \partial C_{2}\right), \\
\llbracket-\epsilon_{0} \nabla_{\mathbf{y}} \phi+\mathbf{p} \rrbracket \cdot \mathbf{n}^{\prime}=\rho_{\mathrm{f}} \quad \text { on } \partial C_{1} \cup \partial C_{2},
\end{gathered}
$$

where $\llbracket \rrbracket$ denotes the jump across the interface and $\mathbf{n}^{\prime}$ is the normal to the boundary.

Each of the terms in the functional (1) has a physical interpretation. The first term penalizes changes in the polarization and thus is interpreted as the energy of forming a ferroelectric domain wall. The second term, the stored energy density, is the energy cost that the crystal must pay if the distortion and polarization deviate from the preferred states at that temperature; thus this builds in the information that the crystal prefers certain spontaneous distortion and spontaneous polarization at a given temperature. The third is the potential energy of the applied electric field, and this enforces the desire of the polarization to align with the applied electric field. The fourth is of course the potential energy of the dead-loading device. The final term is 
the electrostatic self-energy associated with the electric field generated by the ferroelectric crystal.

The variational principle (1) may be justified by considering the total energy of the ferroelectric-conductor system described in $\S 2.1$ :

$$
\begin{aligned}
\overline{\mathcal{E}}(\mathbf{y}, \mathbf{p})= & \int_{\Omega}\left[\frac{1}{2} \nabla_{\mathbf{x}} \mathbf{p} \cdot \mathbf{A} \nabla_{\mathbf{x}} \mathbf{p}+W\left(\nabla_{\mathbf{x}} \mathbf{y}, \mathbf{p}, \theta\right)\right] \mathrm{d} \mathbf{x}-\int_{\partial \Omega} \mathbf{t}_{0} \cdot \mathbf{y} \mathrm{d} S_{\mathbf{x}} \\
& +\frac{\epsilon_{0}}{2} \int_{\mathbb{R}^{3}}\left|\nabla_{\mathbf{y}} \bar{\phi}\right|^{2} \mathrm{~d} \mathbf{y}-\hat{\phi} \int_{\partial C_{2}} \bar{\rho}_{\mathrm{f}} \mathrm{d} S,
\end{aligned}
$$

where the free charge density $\bar{\rho}_{\mathrm{f}}: \partial C_{1} \cup \partial C_{2} \rightarrow \mathbb{R}$ and electric potential $\bar{\phi}: \mathbb{R}^{3} \rightarrow \mathbb{R}$ are obtained by solving Maxwell's equation

$$
\nabla_{\mathbf{y}} \cdot\left(-\epsilon_{0} \nabla_{\mathbf{y}} \bar{\phi}+\mathbf{p} \chi_{\mathbf{y}(\Omega)}\right)=\bar{\rho}_{\mathrm{f}} \quad \text { on } \mathbb{R}^{3}
$$

subject to

$$
\nabla_{\mathbf{y}} \bar{\phi}=\mathbf{0} \quad \text { on } C_{1}, \int_{\partial C_{1}} \frac{\partial \bar{\phi}}{\partial n} \mathrm{~d} S=-\frac{Q}{\epsilon_{0}}, \bar{\phi}=\hat{\phi} \text { on } C_{2}, \bar{\phi} \rightarrow 0 \text { as }|\mathbf{y}| \rightarrow \infty
$$

Let $\phi_{0}$ be the electric field potential and $\rho_{0}: \partial C_{1} \cup \partial C_{2} \rightarrow \mathbb{R}$ be the free charge density that would be present if the ferroelectric were absent. They are obtained by solving Maxwell's equation

$$
-\epsilon_{0} \nabla_{\mathbf{y}}^{2} \phi_{0}=\rho_{0} \quad \text { on } \mathbb{R}^{3}
$$

subject to the boundary conditions (6) with $\bar{\phi}$ replaced by $\phi_{0}$. Now set

$$
\phi=\bar{\phi}-\phi_{0}, \quad \rho_{\mathrm{f}}=\bar{\rho}_{\mathrm{f}}-\rho_{0}, \quad \mathbf{e}_{0}=-\nabla_{\mathbf{y}} \phi_{0} .
$$

Then, it follows from the linearity of Maxwell's equation that $\phi: \mathbb{R}^{3} \rightarrow \mathbb{R}$ and $\rho_{\mathrm{f}}: \partial C_{1} \cup \partial C_{2} \rightarrow \mathbb{R}$ satisfy equation (2) subject to equation (3). Now note that

$$
\begin{aligned}
\epsilon_{0} \int_{\mathbb{R}^{3}} \nabla_{\mathbf{y}} \phi \cdot \nabla_{\mathbf{y}} \phi_{0} \mathrm{~d} \mathbf{y} & =\epsilon_{0} \int_{\mathbb{R}^{3}}\left[\nabla_{\mathbf{y}} \cdot\left(\phi_{0} \nabla_{\mathbf{y}} \phi\right)-\phi_{0} \nabla_{\mathbf{y}}^{2} \phi\right] \mathrm{d} \mathbf{y} \\
& =-\int_{\mathbb{R}^{3}} \phi_{0}\left[\nabla_{\mathbf{y}} \cdot\left(\mathbf{p} \chi_{\mathbf{y}(\Omega)}\right)-\rho_{\mathrm{f}}\right] \mathrm{d} \mathbf{y} \\
& =\int_{\mathbb{R}^{3}}\left(\nabla_{\mathbf{y}} \phi_{0} \cdot \mathbf{p} \chi_{\mathbf{y}(\Omega)}+\rho_{\mathrm{f}} \phi_{0}\right) \mathrm{d} \mathbf{y} \\
& =-\int_{\mathbf{y}(\Omega)} \mathbf{e}_{0} \cdot \mathbf{p} \mathrm{d} \mathbf{y}+\int_{\partial C_{1}} \rho_{\mathrm{f}} \phi_{0} \mathrm{~d} S+\int_{\partial C_{2}} \rho_{\mathrm{f}} \phi_{0} \mathrm{~d} S \\
& =-\int_{\mathbf{y}(\Omega)} \mathbf{e}_{0} \cdot \mathbf{p} \mathrm{d} \mathbf{y}+\hat{\phi} \int_{\partial C_{2}} \rho_{\mathrm{f}} \mathrm{d} S,
\end{aligned}
$$

where we have used the divergence theorem and Maxwell's equations (2) and (3) repeatedly. Substituting equations (8) and (9) into equation (4), we have

$$
\begin{aligned}
\overline{\mathcal{E}}(\mathbf{y}, \mathbf{p})= & \int_{\Omega}\left[\frac{1}{2} \nabla_{\mathbf{x}} \mathbf{p} \cdot \mathbf{A} \nabla_{\mathbf{x}} \mathbf{p}+W\left(\nabla_{\mathbf{x}} \mathbf{y}, \mathbf{p}, \theta\right)-\mathbf{e}_{0} \cdot \mathbf{p}\left(\operatorname{det} \nabla_{\mathbf{x}} \mathbf{y}\right)\right] \mathrm{d} \mathbf{x}-\int_{\partial \Omega} \mathbf{t}_{0} \cdot \mathbf{y} \mathrm{d} S_{\mathbf{x}} \\
& +\frac{\epsilon_{0}}{2} \int_{\mathbb{R}^{3}}\left|\nabla_{\mathbf{y}} \phi\right|^{2} \mathrm{~d} \mathbf{y}+\frac{\epsilon_{0}}{2} \int_{\mathbb{R}^{3}}\left|\nabla_{\mathbf{y}} \phi_{0}\right|^{2} \mathrm{~d} \mathbf{y}-\hat{\phi} \int_{\partial C_{2}} \rho_{0} \mathrm{~d} S .
\end{aligned}
$$


Note that the sum of the last two terms of equation (10) (the energy of the conductor system in the absence of the ferroelectric crystal) does not depend on $\mathbf{y}$ and $\mathbf{p}$. Therefore removing them from the energy does not change the variational principle, but yields the functional (1).

Although we have justified equation (1) in the special case of the ferroelectricconductor system shown in figure 1, the functional is still valid if the applied field is created by some other arrangement (using for example other dielectrics) provided that we interpret $\mathbf{e}_{0}$ as the electric field that would be present if the ferroelectric crystal were absent, and Maxwell's equation (2) were solved using appropriate boundary conditions.

We make two comments for future use. First, we can rewrite the last term in the energy using Maxwell's equations as

$$
\frac{1}{2} \int_{\mathbf{y}(\Omega)} \mathbf{p}(\mathbf{y}) \cdot \nabla_{\mathbf{y}} \phi(\mathbf{y}) \mathrm{d} \mathbf{y} .
$$

Second, we can replace Maxwell's equations by the following variational principle:

$$
\min \left(\int_{\mathbb{R}^{3}}\left|-\epsilon_{0} \nabla_{\mathbf{y}} \phi+\mathbf{p} \chi_{\mathbf{y}(\Omega)}\right|^{2} \mathrm{~d} \mathbf{y}\right)
$$

over all $\phi$ that satisfy equation (3).

\subsection{Multiwell structure}

Let us now turn to the basic properties of the stored energy density $W$. We require it to possess the following two fundamental properties:

(i) Frame indifference. We assume that $W$ is objective; that is it is unaffected by a change in frame or an additional rigid body rotation of the current configuration:

$$
W(\mathbf{Q F}, \mathbf{Q p}, \theta)=W(\mathbf{F}, \mathbf{p}, \theta)
$$

for all $\mathbf{Q} \in \mathrm{SO}$ (3), for all $\mathbf{F} \in \mathbb{M}^{3 \times 3}$ with $\operatorname{det} \mathbf{F}>0$ and for all $\mathbf{p} \in \mathbb{R}^{3}$.

(ii) Material symmetry. We assume that $W$ reflects the crystalline symmetry of the ferroelectric crystal. In other words, we assume that a change in reference configuration by a member of the point group $\mathcal{P}_{0}$ (which is defined as the group of rotations that map the reference lattice back to itself) leaves $W$ invariant:

$$
W(\mathbf{F R}, \mathbf{p}, \theta)=W(\mathbf{F}, \mathbf{p}, \theta)
$$

for all $\mathbf{R} \in \mathcal{P}^{0}$, for all $\mathbf{F} \in \mathbb{M}^{3 \times 3}$ with $\operatorname{det} \mathbf{F}>0$ and for all $\mathbf{p} \in \mathbb{R}^{3}$.

In considering these invariances, it is useful to recall that the polarization is defined in the current configuration. For future use we combine these relations and note that

$$
W\left(\mathbf{Q R} \mathbf{R}^{\mathrm{T}} \mathbf{F R}, \mathbf{Q} \mathbf{R}^{\mathrm{T}} \mathbf{p}, \theta\right)=W(\mathbf{F}, \mathbf{p}, \theta)
$$

for all $\mathbf{R} \in \mathcal{P}^{0}$, for all $\mathbf{Q} \in \mathrm{SO}(3)$ and for all $\mathbf{F} \in \mathbb{M}^{3 \times 3}$ with $\operatorname{det} \mathbf{F}>0$ and for all $\mathbf{p} \in \mathbb{R}^{3}$.

Ferroelectric crystals are non-polar above the Curie temperature with a preferred (stress-free) lattice structure but are spontaneously polarized below the Curie tem- 
perature with a spontaneous lattice distortion. Our stored energy function $W$ should reflect this. We discuss this in detail for the special case of $\mathrm{BaTiO}_{3}$, which is one of the most widely studied ferroelectric materials. This discussion can be adapted to other ferroelectrics; in particular we comment on lead zirconate titanate (PZT) at the end of this section.

$\mathrm{BaTiO}_{3}$ undergoes a series of three first-order phase transformations as summarized in figure 2. Above $120^{\circ} \mathrm{C}$ it is non-polar and has a cubic perovskite $\mathrm{ABO}_{3}$ structure as shown at the bottom left of figure 2 . Between 5 and $120^{\circ} \mathrm{C}$, it has a tetragonal lattice structure as shown at the bottom right of figure 2 and is spontaneously polarized along the pseudocubic $\langle 100\rangle$ axis. It is spontaneously polarized in a pseudocubic $\langle 110\rangle$ direction and has an orthorhombic structure between -90 and $5^{\circ} \mathrm{C}$ and finally is spontaneously polarized in a pseudocubic $\langle 111\rangle$ direction and has a rhombohedral structure below $-90^{\circ} \mathrm{C}$.

Now consider a single crystal of undistorted $\mathrm{BaTiO}_{3}$ at the Curie temperature of $120^{\circ} \mathrm{C}$ and choose this as the reference configuration. First, note that $\mathcal{P}^{0}$ is the point group of the cubic lattice or the 24 proper rotations that map the cube back into itself (see appendix A). Next, recall from $\S 2.1$ that we can represent the different states of the crystal by the pair $(\mathbf{F}, \mathbf{p})$ where $\mathbf{F}$ is the deformation gradient with respect to the reference configuration and $\mathbf{p}$ is the polarization. Thus, the cubic state is described by $(\mathbf{I}, \mathbf{0})$, the tetragonal state by $\left(\mathbf{U}_{1}^{\mathrm{t}}, \mathbf{p}_{1}^{\mathrm{t}}\right)$, the orthorhombic state by $\left(\mathbf{U}_{1}^{\mathrm{o}}, \mathbf{p}_{1}^{\mathrm{o}}\right)$ and the rhombohedral state by $\left(\mathbf{U}_{1}^{\mathrm{r}}, \mathbf{p}_{1}^{\mathrm{r}}\right)$ where $\mathbf{I}$ is the identity matrix, $\mathbf{U}_{1}^{\mathrm{t}}$ is the matrix that maps the cubic unit cell to the tetragonal (i.e. unit cell at the bottom left of figure 2 to that at the bottom right) and so forth. These depend on temperature (owing to thermal expansion); we ignore this here for simplicity but note that the theory can be easily be modified to account for it.

Since different phases are stable at different temperatures, we conclude that the stored energy density $W$ must have different minimizers at different temperatures as shown schematically in figure 3. In particular, (I, 0) should minimize the energy above $120^{\circ} \mathrm{C},\left(\mathbf{U}_{1}^{t}, \mathbf{p}_{1}^{t}\right)$ between 5 and $120^{\circ} \mathrm{C}$ and so forth:

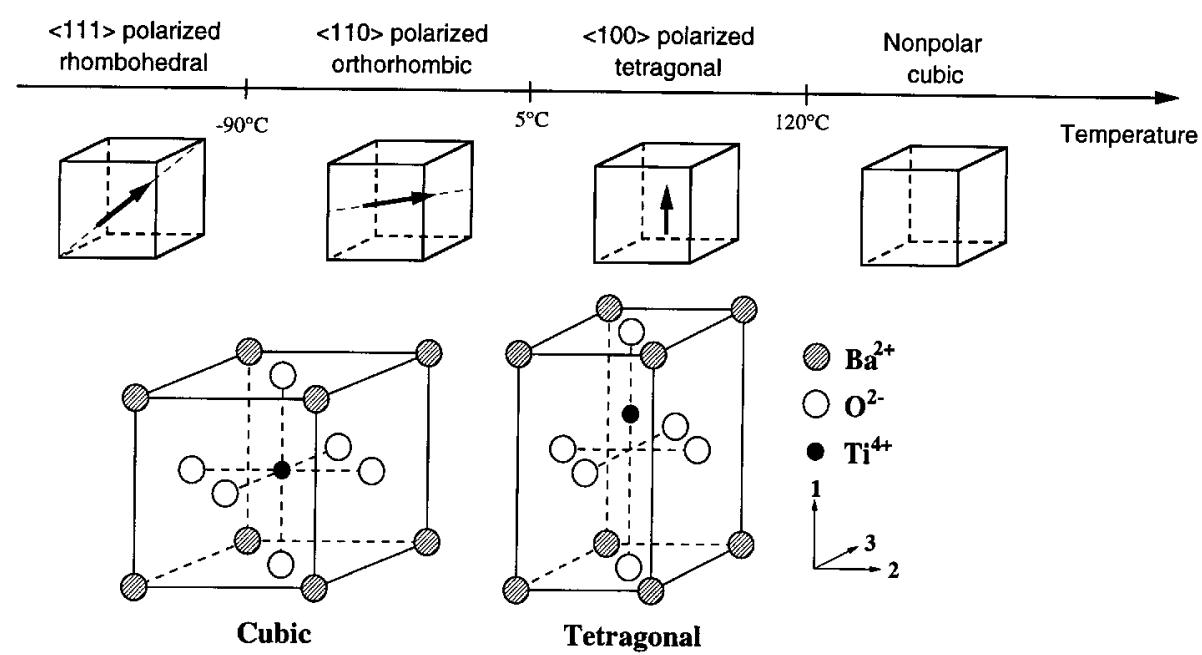

Figure 2. The spontaneous polarization and lattice structure of $\mathrm{BaTiO}_{3}$ at different temperatures. 


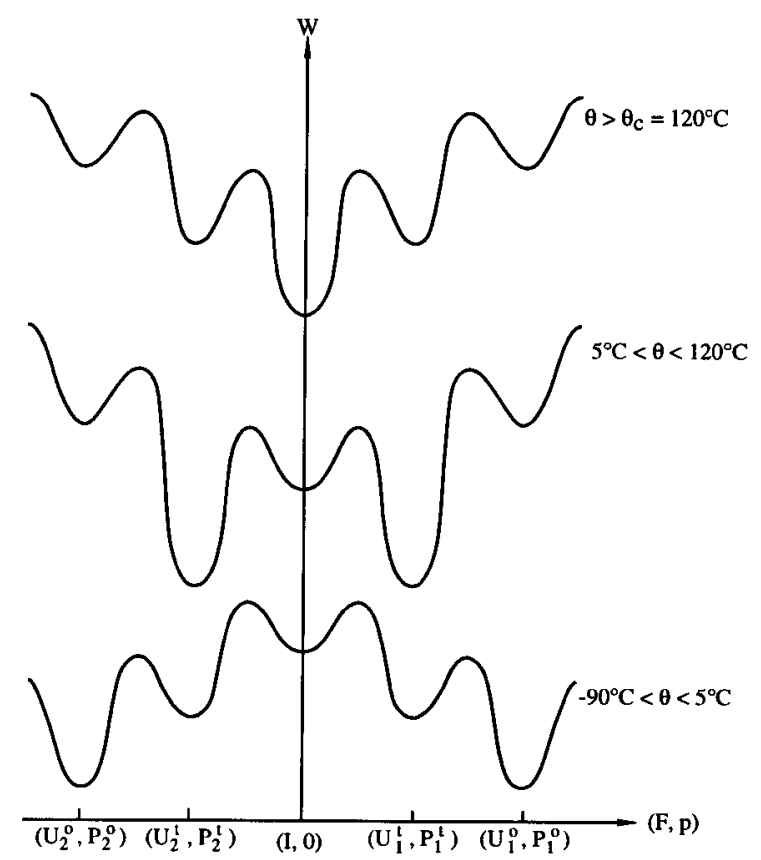

Figure 3. A schematic view of the stored energy density $W$. Here we do not show the $\langle 111\rangle$ polarized states, and show only two of the six (12) possible $\langle 100\rangle(\langle 110\rangle)$ polarized states.

$$
\begin{aligned}
W(\mathbf{I}, \mathbf{0}, \theta) \leqslant W(\mathbf{F}, \mathbf{p}, \theta) & \forall(\mathbf{F}, \mathbf{p}), 120^{\circ} \mathrm{C} \leqslant \theta, \\
W\left(\mathbf{U}_{1}^{\mathrm{t}}, \mathbf{p}_{1}^{\mathrm{t}}, \theta\right) \leqslant W(\mathbf{F}, \mathbf{p}, \theta) & \forall(\mathbf{F}, \mathbf{p}), 5^{\circ} \mathrm{C} \leqslant \theta \leqslant 120^{\circ} \mathrm{C}, \\
W\left(\mathbf{U}_{1}^{\mathrm{o}}, \mathbf{p}_{1}^{\mathrm{o}}, \theta\right) \leqslant W(\mathbf{F}, \mathbf{p}, \theta) & \forall(\mathbf{F}, \mathbf{p}),-90^{\circ} \mathrm{C} \leqslant \theta \leqslant 5^{\circ} \mathrm{C}, \\
W\left(\mathbf{U}_{1}^{\mathrm{r}}, \mathbf{p}_{1}^{\mathrm{r}}, \theta\right) \leqslant W(\mathbf{F}, \mathbf{p}, \theta) & \forall(\mathbf{F}, \mathbf{p}), \theta \leqslant-90^{\circ} \mathrm{C} .
\end{aligned}
$$

However, frame indifference and material symmetry (as summarized in equation (15)) imply the existence of minimizers other than those described above. For example, between 5 and $120^{\circ} \mathrm{C}$, we conclude that $W$ is minimized on the set

$$
\left\{(\mathbf{G}, \mathbf{q}): \mathbf{G}=\mathbf{Q} \mathbf{U}_{1}^{\mathrm{t}} \mathbf{R}, \mathbf{q}=\mathbf{Q} \mathbf{p}_{1}^{\mathrm{t}}, \forall \mathbf{Q} \in \mathrm{SO}(3), \forall \mathbf{R} \in \mathcal{P}^{0}\right\}
$$

These sets have a very simple characterization based on which we conclude the following.

(i) Non-polar cubic wells. Above $120^{\circ} \mathrm{C}, \mathrm{W}$ is minimized on the set

$$
\mathcal{W}^{c}=\{(\mathbf{Q}, \mathbf{0}): \mathbf{Q} \in \mathrm{SO}(3)\} .
$$

A rigid rotation of the crystal still keeps it in the cubic phase; so the cubic phase corresponds to the 'well' of all rigid rotations of the reference configuration.

(ii) $\langle 100\rangle$ tetragonal wells. Between 5 and $120^{\circ} \mathrm{C}, W$ is minimized on the set

$$
\mathcal{W}^{t}=\left\{\left(\mathbf{Q U}_{i}^{\mathrm{t}}, \pm \mathbf{Q} \mathbf{p}_{i}^{\mathrm{t}}\right): \mathbf{Q} \in \mathbf{S O}(3), i=1,2,3\right\},
$$


where

$$
\begin{gathered}
\mathbf{p}_{1}^{\mathrm{t}}=\xi^{\mathrm{t}}\{1,0,0\}, \quad \mathbf{p}_{2}^{\mathrm{t}}=\xi^{\mathrm{t}}\{0,1,0\}, \quad \mathbf{p}_{3}^{\mathrm{t}}=\xi^{\mathrm{t}}\{0,0,1\}, \\
\left.\mathbf{U}_{1}^{t}=\left(\begin{array}{ccc}
\eta_{2}^{\mathrm{t}} & 0 & 0 \\
0 & \eta_{1}^{\mathrm{t}} & 0 \\
0 & 0 & \eta_{1}^{\mathrm{t}}
\end{array}\right), \quad \mathbf{U}_{2}^{\mathrm{t}}=\left(\begin{array}{ccc}
\eta_{1}^{\mathrm{t}} & 0 & 0 \\
0 & \eta_{2}^{\mathrm{t}} & 0 \\
0 & 0 & \eta_{1}^{\mathrm{t}}
\end{array}\right), \quad \mathbf{U}_{3}^{t}=\left(\begin{array}{ccc}
\eta_{1}^{\mathrm{t}} & 0 & 0 \\
0 & \eta_{1}^{\mathrm{t}} & 0 \\
0 & 0 & \eta_{2}^{\mathrm{t}}
\end{array}\right), \quad, \quad 18\right)
\end{gathered}
$$

and the measured values for $\mathrm{BaTiO}_{3}$ are $\xi^{\mathrm{t}}=0.26 \mathrm{C} \mathrm{m}^{-2}, \eta_{2}^{\mathrm{t}}=1.0067$ and $\eta_{1}^{\mathrm{t}}=0.9958$ (Mitsui et al. 1969). Note that, according to material symmetry, the crystal can be polarized in six equivalent $\langle 100\rangle$ directions; thus there are six symmetry-related variants of the tetragonal phase and this corresponds to the fact that $\mathcal{W}^{t}$ consists of six wells. This set is shown in figure 4 .

(iii) $\langle 110\rangle$ orthorhombic wells. Between -90 and $5^{\circ} \mathrm{C}, W$ is minimized on the set

$$
\mathcal{W}^{0}=\left\{\left(\mathbf{Q} \mathbf{U}_{i}^{\mathrm{o}}, \pm \mathbf{Q} \mathbf{p}_{i}^{\mathrm{o}}\right): \mathbf{Q} \in \mathrm{SO}(3), i=1, \ldots, 6\right\}
$$

where

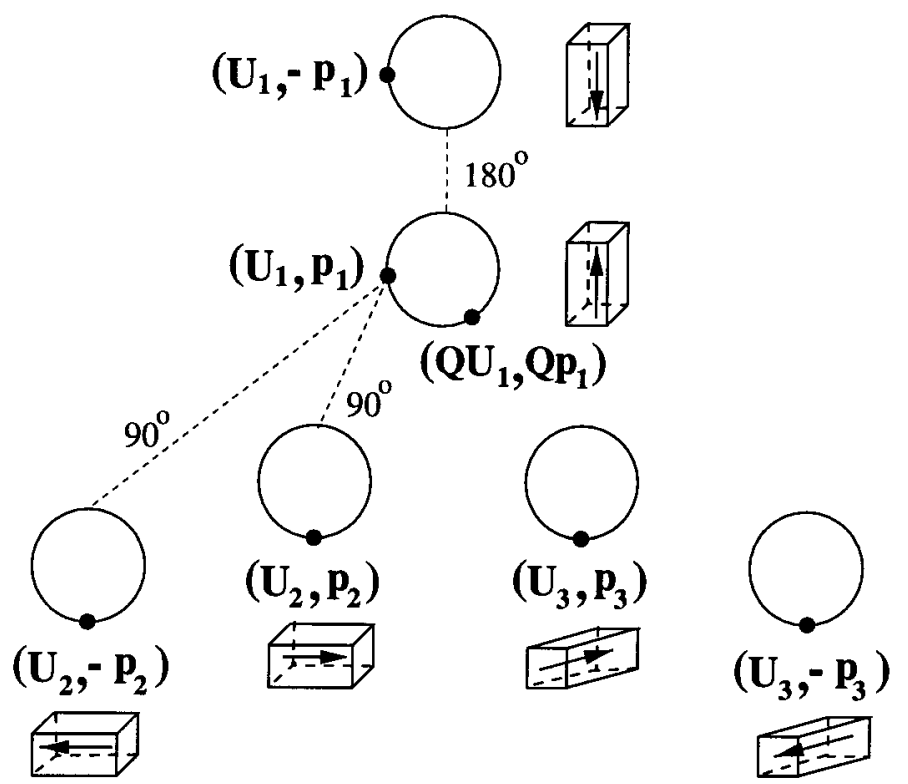

Figure 4. Energy wells $\mathcal{W}^{\mathrm{t}}$ and domain walls of $\mathrm{BaTiO}_{3}$ in its tetragonal phase. Let the plane of the paper be the set of all states $(\mathbf{F}, \mathbf{p}) .\left(\mathbf{U}_{1}, \mathbf{p}_{1}\right),\left(\mathbf{U}_{1},-\mathbf{p}_{1}\right),\left(\mathbf{U}_{2}, \mathbf{p}_{2}\right), \ldots$ are the six crystallographically equivalent polarized states or variants, and the open circles represent the states obtained from any of these states $\left(\mathbf{U}_{i}, \mathbf{p}_{i}\right)$ by a rigid-body rotation (change in observer frame). The possible domain walls are shown as broken lines; any variant can form a $180^{\circ}$ domain wall with the variant with the same stretch and opposite polarization, and one $90^{\circ}$ domain wall with exactly one state of each of the other variants. Some of the lines are not shown to keep the figure reasonably comprehensible. 


$$
\begin{aligned}
& \mathbf{p}_{1}^{\mathrm{o}}=\xi^{\mathrm{o}}\{1,1,0\}, \quad \mathbf{p}_{2}^{\mathrm{o}}=\xi^{\mathrm{o}}\{-1,1,0\}, \\
& \mathbf{U}_{1}^{\mathrm{o}}=\left(\begin{array}{ccc}
\alpha^{\mathrm{o}} & \delta^{\mathrm{o}} & 0 \\
\delta^{\mathrm{o}} & \alpha^{\mathrm{o}} & 0 \\
0 & 0 & \beta^{\mathrm{o}}
\end{array}\right), \quad \mathbf{U}_{2}^{\mathrm{o}}=\left(\begin{array}{ccc}
\alpha^{\mathrm{o}} & -\delta^{\mathrm{o}} & 0 \\
-\delta^{\mathrm{o}} & \alpha^{\mathrm{o}} & 0 \\
0 & 0 & \beta^{\mathrm{o}}
\end{array}\right) \text {, } \\
& \mathbf{p}_{3}^{\mathrm{o}}=\xi^{\mathrm{o}}\{1,0,1\}, \quad \mathbf{p}_{4}^{\mathrm{o}}=\xi^{\mathrm{o}}\{-1,0,1\} \\
& \mathbf{U}_{3}^{\mathrm{o}}=\left(\begin{array}{ccc}
\alpha^{\mathrm{o}} & 0 & \delta^{\mathrm{o}} \\
0 & \beta^{\mathrm{o}} & 0 \\
\delta^{\mathrm{o}} & 0 & \alpha^{\mathrm{o}}
\end{array}\right), \quad \mathbf{U}_{4}^{\mathrm{o}}=\left(\begin{array}{ccc}
\alpha^{\mathrm{o}} & 0 & -\delta^{\mathrm{o}} \\
0 & \beta^{\mathrm{o}} & 0 \\
-\delta^{\mathrm{o}} & 0 & \alpha^{\mathrm{o}}
\end{array}\right), \\
& \mathbf{p}_{5}^{\mathrm{o}}=\xi^{\mathrm{o}}\{0,1,1\}, \quad \mathbf{p}_{6}^{\mathrm{o}}=\xi^{\mathrm{o}}\{0,-1,1\}, \\
& \mathbf{U}_{5}^{\mathrm{o}}=\left(\begin{array}{ccc}
\beta^{\mathrm{o}} & 0 & 0 \\
0 & \alpha^{\mathrm{o}} & \delta^{\mathrm{o}} \\
0 & \delta^{\mathrm{o}} & \alpha^{\mathrm{o}}
\end{array}\right), \quad \mathbf{U}_{6}^{\mathrm{o}}=\left(\begin{array}{ccc}
\beta^{\mathrm{o}} & 0 & 0 \\
0 & \alpha^{\mathrm{o}} & -\delta^{\mathrm{o}} \\
0 & -\delta^{\mathrm{o}} & \alpha^{\mathrm{o}}
\end{array}\right) \text {, }
\end{aligned}
$$

and the measured values for $\mathrm{BaTiO}_{3}$ are $\xi^{\mathrm{o}}=0.21 \mathrm{C} \mathrm{m}^{-2}, \alpha^{\mathrm{o}}=1.00329$, $\beta^{\mathrm{o}}=0.9975$ and $\delta^{\mathrm{o}}=0.00115$ (Mitsui et al. 1969). Note that, according to material symmetry, the crystal can be polarized in 12 equivalent $\langle 110\rangle$ directions; thus there are 12 symmetry-related variants of the orthorhombic phase and $\mathcal{W}^{\circ}$ consists of 12 wells. This set is shown in figure 5.

(iv) $\langle 111\rangle$ rhombohedral wells. Below $-90^{\circ} \mathrm{C}, W$ is minimized on the set

$$
\mathcal{W}^{\mathrm{r}}=\left\{\left(\mathbf{Q} \mathbf{U}_{i}^{\mathrm{r}}, \pm \mathbf{Q} \mathbf{p}_{i}^{\mathrm{r}}\right): \mathbf{Q} \in \operatorname{SO}(3), i=1, \ldots, 4\right\}
$$

where

$$
\begin{array}{ll}
\mathbf{p}_{1}^{\mathrm{r}}=\xi^{\mathrm{r}}\{1,1,1\}, & \mathbf{p}_{2}^{\mathrm{r}}=\xi^{\mathrm{r}}\{-1,1,1\}, \\
\mathbf{U}_{1}^{\mathrm{r}}=\left(\begin{array}{ccc}
\alpha^{\mathrm{r}} & \delta^{\mathrm{r}} & \delta^{\mathrm{r}} \\
\delta^{\mathrm{r}} & \alpha^{\mathrm{r}} & \delta^{\mathrm{r}} \\
\delta^{\mathrm{r}} & \delta^{\mathrm{r}} & \alpha^{\mathrm{r}}
\end{array}\right), & \mathbf{U}_{2}^{\mathrm{r}}=\left(\begin{array}{ccc}
\alpha^{\mathrm{r}} & -\delta^{\mathrm{r}} & -\delta^{\mathrm{r}} \\
-\delta^{\mathrm{r}} & \alpha^{\mathrm{r}} & \delta^{\mathrm{r}} \\
-\delta^{\mathrm{r}} & \delta^{\mathrm{r}} & \alpha^{\mathrm{r}}
\end{array}\right), \\
\mathbf{p}_{3}^{\mathrm{r}}=\xi^{\mathrm{r}}\{1,1,-1\}, & \mathbf{p}_{4}^{\mathrm{r}}=\xi^{\mathrm{r}}\{1,-1,1\}, \\
\mathbf{U}_{3}^{\mathrm{r}}=\left(\begin{array}{ccc}
\alpha^{\mathrm{r}} & \delta^{\mathrm{r}} & -\delta^{\mathrm{r}} \\
\delta^{\mathrm{r}} & \alpha^{\mathrm{r}} & -\delta^{\mathrm{r}} \\
-\delta^{\mathrm{r}} & -\delta^{\mathrm{r}} & \alpha^{\mathrm{r}}
\end{array}\right), \mathbf{U}_{4}^{\mathrm{r}}=\left(\begin{array}{ccc}
\alpha^{\mathrm{r}} & -\delta^{\mathrm{r}} & \delta^{\mathrm{r}} \\
-\delta^{\mathrm{r}} & \alpha^{\mathrm{r}} & -\delta^{\mathrm{r}} \\
\delta^{\mathrm{r}} & -\delta^{\mathrm{r}} & \alpha^{\mathrm{r}}
\end{array}\right),
\end{array}
$$

and the measured values for $\mathrm{BaTiO}_{3}$ are $\xi^{\mathrm{r}}=0.19 \mathrm{C} \mathrm{m}^{-2}, \alpha^{\mathrm{r}}=0.99999$ and $\delta^{r}=0.00131$ (Mitsui et al. 1969). Note that there are eight equivalent $\langle 111\rangle$ directions and hence $\mathcal{W}^{r}$ has eight wells. This set is shown in figure 6.

PZT, a solid solution of lead titanate $\left(\mathrm{PbTiO}_{3}\right)$ and lead zirconate $\left(\mathrm{PbZrO}_{3}\right)$, is widely used in applications, especially at compositions close to the morphotropic phase boundary. Pure $\mathrm{PbTiO}_{3}$ is cubic and non-polar above the Curie temperature, and $\langle 100\rangle$ polarized tetragonal below it; so the wells at room temperature are given 


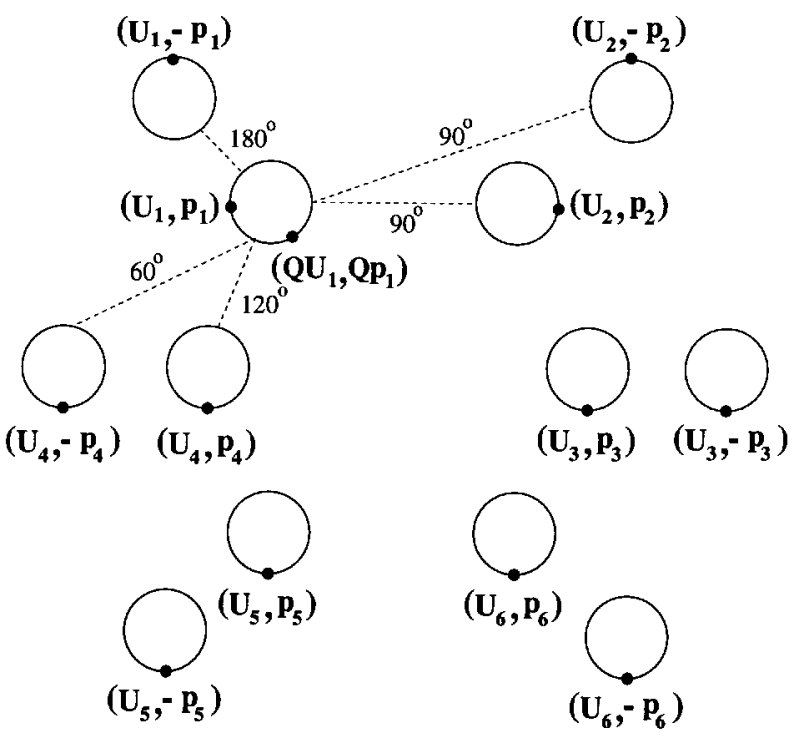

Figure 5. Energy wells $\mathcal{W}^{\circ}$ and domain walls of $\mathrm{BaTiO}_{3}$ in its orthorhombic phase. The symbols have the same meanings as in figure 4 . Any orthorhombic variant can form a $180^{\circ}$ domain wall with the variant with the same stretch and opposite polarization, a $90^{\circ}$ wall with each of the two variants with different stretch but same $c$ axis, and either a $60^{\circ}$ or $120^{\circ}$ wall with each of the other eight variants. Some of the lines are not shown to keep the figure reasonably comprehensible.
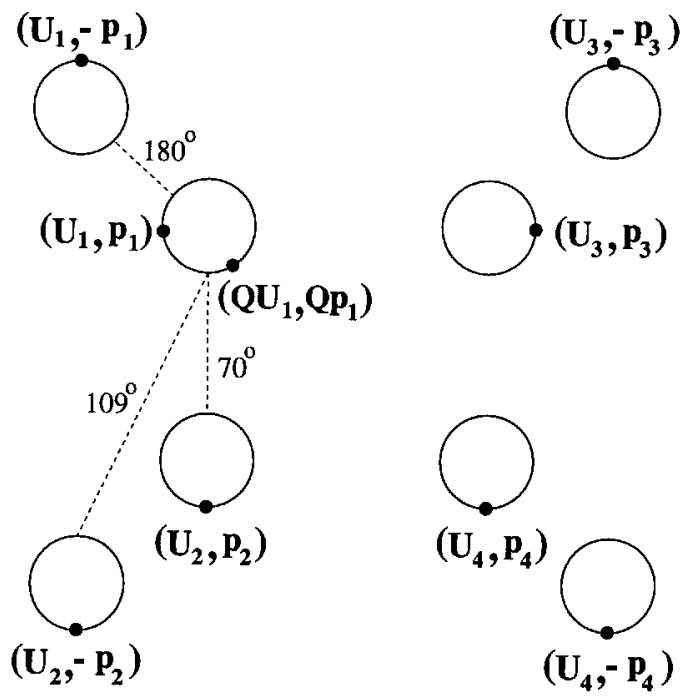

Figure 6. Energy wells $\mathcal{W}^{\mathrm{r}}$ and domain walls of $\mathrm{BaTiO}_{3}$ in its rhombohedral phase. The symbols have the same meanings as in figure 4 . Any rhombohedral variant can form a $180^{\circ}$ domain wall with the variant with the same stretch and opposite polarization, and either a $70^{\circ}$ or a $109^{\circ}$ wall with each of the other six variants. Some of the lines are not shown to keep the figure reasonably comprehensible. 
by equations (17) and (18) with the measured values $\eta_{1}^{\mathrm{t}}=0.9824$ and $\eta_{2}^{\mathrm{t}}=1.0453$ (Mitsui et al. 1969). Pure $\mathrm{PbZrO}_{3}$ is cubic and non-polar above its Curie temperature, and antiferroelectric at room temperature; however, doping it with a small amount of Ti makes it ferroelectric with $\langle 111\rangle$ polarization below the Curie temperature. Therefore the wells for $\mathrm{PbZrO}_{3}$ are given by equations (21) and (22) with measured values $\alpha^{\mathrm{r}}=0.9999, \delta^{\mathrm{r}}=0.00203$ (Mitsui et al. 1969). The solid-solution PZT is $\langle 100\rangle$ polarized tetragonal in the Ti-rich region and $\langle 111\rangle$ polarized rhombohedral in the Zr-rich region, with an exchange of stability at the 'morphotropic phase boundary' (at approximately $\mathrm{Pb}\left(\mathrm{Ti}_{0.45} \mathrm{Zr}_{0.55}\right) \mathrm{O}_{3}$ composition). Therefore at the morphotropic phase boundary the crystal can have either the $\langle 100\rangle$ or the $\langle 111\rangle$ polarization, and hence have both set of wells: the $\langle 100\rangle$ wells (17) and $\langle 111\rangle$ wells (21). The measured values are given by $\eta_{1}^{\mathrm{t}}=1.0138, \eta_{2}^{\mathrm{t}}=1.0327$, $\alpha^{\mathrm{r}}=0.9999$, and $\delta^{\mathrm{r}}=0.0029$ around the morphotropic composition (Mitsui et al. 1969).

\section{§3. DOMAIn PaTterns}

In this section we show that the multiwell structure of the stored energy density $W$ gives rise to minimizers that consist of domains of differently oriented polarization. Further, these domains form very characteristic patterns. In this section we study domain patterns in a large ferroelectric single crystal held at a constant temperature in the absence of external loadings, that is when $\mathbf{e}_{0}=\mathbf{0}$ and $\mathbf{t}_{0}=\mathbf{0}$.

Let us go back to the total energy of the single crystal described in equation (1). Recall that the first term is the energy associated with spatial changes in polarization or domain walls. If this term is small or, equivalently, if the size of the crystal under consideration is large compared with the square root of the largest eigenvalue of the matrix $\mathbf{A}$, then the crystal can form many domains if it chooses to, and this term does not influence the overall domain pattern and macroscopic behaviour. Thus, it can be neglected in a large crystal. This statement can be made rigorous by adopting the arguments of DeSimone (1993).

Thus we are led to the problem of minimizing the energy

$$
\hat{\mathcal{E}}(\mathbf{y}, \mathbf{p})=\int_{\Omega} W\left(\nabla_{\mathbf{x}} \mathbf{y}, \mathbf{p}\right) \mathrm{d} \mathbf{x}+\frac{\epsilon_{0}}{2} \int_{\mathbb{R}^{3}}\left|\nabla_{\mathbf{y}} \phi\right|^{2} \mathrm{~d} \mathbf{y},
$$

where we obtain the electric potential $\phi$ by solving Maxwell's equation (2). Above, we have dropped temperature from the notation. Further, since it is held constant, we assume that $W$ is minimized on a suitable set $\mathcal{W}$ and that its minimum value is zero without any loss of generality.

Note that each of these terms in (23) is positive, and thus to minimize it we need to minimize them individually. The first term thus requires that

$$
\left(\nabla_{\mathbf{x}} \mathbf{y}(\mathbf{x}), \mathbf{p}(\mathbf{y}(\mathbf{x}))\right) \in \mathcal{W}
$$

at each point $\mathbf{x} \in \Omega$ where $\mathcal{W}$ is the set of appropriate energy wells from among equations (16), (17), (19) or (21). The second term requires that the electric field be zero, and this in turn requires that $\mathbf{p}$ be divergence free:

$$
\nabla_{\mathbf{y}} \cdot \mathbf{p}(\mathbf{y}(\mathbf{x}))=0 \quad \text { on } \mathbb{R}^{3}
$$




\subsection{Domain Walls}

Consider a piecewise homogeneous state as shown in figure 7 with deformation gradient and polarization taking values $\left(\mathbf{F}^{+}, \mathbf{p}^{+}\right)$in one part of the crystal and $\left(\mathbf{F}^{-}, \mathbf{p}^{-}\right)$in the other. Assume that the two parts are separated by a plane with normal $\hat{\mathbf{n}}$ in the reference configuration. We assume that the deformation is continuous so that the crystal does not break apart. Then, the deformation gradients $\mathbf{F}^{+}$ and $\mathbf{F}^{-}$on the two sides cannot be arbitrary but must satisfy the Hadamard jump condition (for example Gurtin (1981)):

$$
\mathbf{F}^{+}-\mathbf{F}^{-}=\mathbf{a}^{\prime} \otimes \hat{\mathbf{n}}
$$

for some vector $\mathbf{a}^{\prime}$ where $\mathbf{a}^{\prime} \otimes \hat{\mathbf{n}}$ is the matrix with components $\left(\mathbf{a}^{\prime} \otimes \hat{\mathbf{n}}\right)_{i j}=a_{i}^{\prime} \hat{n}_{j}$ and $\mathbf{a}^{\prime}=\left(a_{i}^{\prime}\right), \hat{\mathbf{n}}=\left(\hat{n}_{i}\right)$. Further, the interface has a normal

$$
\mathbf{n}^{\prime}=\frac{\left(\mathbf{F}^{+}\right)^{-\mathrm{T}} \hat{\mathbf{n}}}{\left|\left(\mathbf{F}^{+}\right)^{-\mathrm{T}} \hat{\mathbf{n}}\right|}=\frac{\left(\mathbf{F}^{-}\right)^{-\mathrm{T}} \hat{\mathbf{n}}}{\left|\left(\mathbf{F}^{-}\right)^{-\mathrm{T}} \hat{\mathbf{n}}\right|}
$$

in the current configuration.

We now examine the necessary conditions for such a piecewise homogeneous configuration to be an energy minimizer. According to equation (24) we require that $\left(\mathbf{F}^{ \pm}, \mathbf{p}^{ \pm}\right) \in \mathcal{W}$ or, equivalently,

$$
\begin{array}{ll}
\mathbf{F}^{+}=\mathbf{Q}^{+} \mathbf{U}_{j}, & \mathbf{p}^{+}= \pm \mathbf{Q}^{+} \mathbf{p}_{j}, \\
\mathbf{F}^{-}=\mathbf{Q}^{-} \mathbf{U}_{i}, & \mathbf{p}^{-}= \pm \mathbf{Q}^{-} \mathbf{p}_{i},
\end{array}
$$

for some $\mathbf{Q}^{ \pm} \in \mathrm{SO}(3)$ and for some $i, j$. Further according to equation (25) interpreted in the weak form, we require that

$$
\left(\mathbf{p}^{+}-\mathbf{p}^{-}\right) \cdot \mathbf{n}^{\prime}=0 .
$$

Setting $\mathbf{Q}=\left(\mathbf{Q}^{-}\right)^{\mathrm{T}} \mathbf{Q}^{+}$and $\mathbf{a}=\left(\mathbf{Q}^{-}\right)^{\mathrm{T}} \mathbf{a}^{\prime}$ we conclude the following from equations (26)-(29). We can form an interface between variants $i$ and $j$ if and only if we satisfy

$$
\begin{array}{r}
\mathbf{Q U _ { j }}-\mathbf{U}_{i}=\mathbf{a} \otimes \hat{\mathbf{n}}, \\
\left(\kappa \mathbf{Q} \mathbf{p}_{j}-\mathbf{p}_{i}\right) \cdot \mathbf{U}_{i}^{-\mathrm{T}} \hat{\mathbf{n}}=0
\end{array}
$$

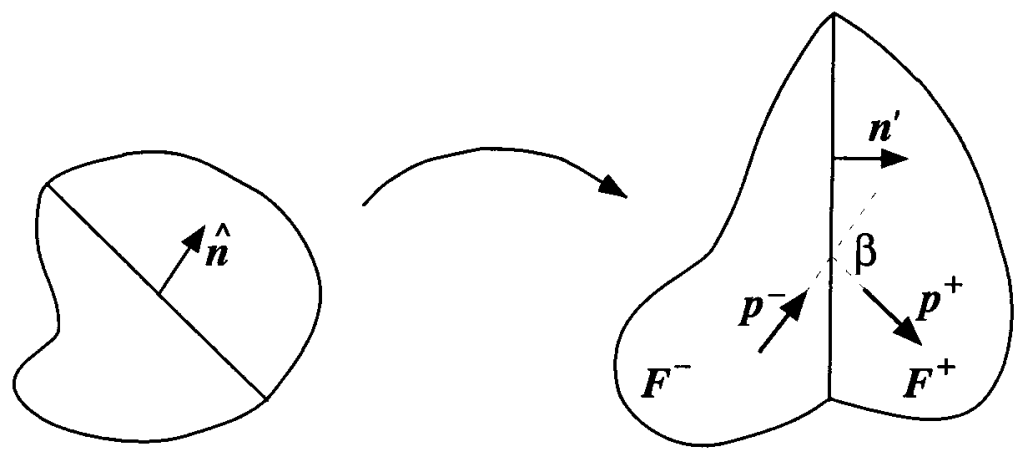

Figure 7. A piecewise homogeneous state in the reference (left) and current (right) configuration. 
for some $\mathbf{Q} \in \mathrm{SO}(3), \mathbf{a} \in \mathbb{R}^{3}, \hat{\mathbf{n}} \in \mathbb{R}^{3}, \kappa= \pm 1$. The first of these equations, the mechanical compatibility equation (30), is the same as the 'twinning equation' in martensites and guarantees the mechanical integrity of the interface. The second of these, the electrical compatibility equation (31), ensures that there are no bound charges on the interface.

Let us now examine whether we can solve these equations for a given pair $\left(\mathbf{U}_{i}, \mathbf{p}_{i}\right)$ and $\left(\mathbf{U}_{j}, \mathbf{p}_{j}\right)$. Ball and James have given the most general conditions for solving the twinning equation (30) for a given pair $\left(\mathbf{U}_{i}, \mathbf{U}_{j}\right)$. They show that there are at most two solutions $\left(\mathbf{Q}^{\mathrm{I}}, \mathbf{a}^{\mathrm{I}}, \hat{\mathbf{n}}^{\mathrm{I}}\right)$ and $\left(\mathbf{Q}^{\mathrm{II}}, \mathbf{a}^{\mathrm{II}}, \hat{\mathbf{n}}^{\mathrm{II}}\right)$. In other words, the twinning equation completely determines the unknown quantities. Thus the system of equations (30) and (31) is overdetermined and generically has no solution.

However, it is possible to have solutions under certain very special conditions on $\left(\mathbf{U}_{i}, \mathbf{p}_{i}\right)$ and $\left(\mathbf{U}_{j}, \mathbf{p}_{j}\right)$. It turns out that symmetry forces the satisfaction of these conditions in many materials of interest as demonstrated in the following lemma. A similar result has been independently derived by DeSimone and James (2001) in the context of magnetostriction. Thus the existence of low-energy domain walls is a consequence of symmetry.

Lemma 1 (classification of domain walls): Let $\left(\mathbf{U}_{i}, \mathbf{p}_{i}\right)$ and $\left(\mathbf{U}_{j}, \mathbf{p}_{j}\right)$ be given

(a) Suppose that $i=j$. Then equations (30) and (31) are both satisfied by $\mathbf{Q}=\mathbf{I}$, $\mathbf{a}=\mathbf{0}, \kappa=-1$ and any $\hat{\mathbf{n}}$ that satisfies $\hat{\mathbf{n}} \cdot \mathbf{U}_{i}^{-1} \mathbf{p}_{i}=0$. This gives a $180^{\circ}$ domain wall. Note that $\kappa=+1$ gives a degenerate solution since it implies that there is no difference between the two sides.

(b) Suppose that $i \neq j$. Further suppose that the two sides are related by a twofold rotation, that is let $\mathbf{U}_{i}$ and $\mathbf{U}_{j}$ satisfy

$$
\mathbf{U}_{j}=\mathbf{R}^{\mathrm{T}} \mathbf{U}_{i} \mathbf{R}, \quad \mathbf{p}_{j}=\mathbf{R}^{\mathrm{T}} \mathbf{p}_{i},
$$

for some twofold rotation $\mathbf{R}=-\mathbf{I}+2 \hat{\mathbf{v}} \otimes \hat{\mathbf{v}}$ with axis $\hat{\mathbf{v}}$. Then, equations (30) and (31) have two solutions given by

$\hat{\mathbf{n}}^{(\mathrm{I})}=\hat{\mathbf{v}}, \quad \mathbf{a}^{(\mathrm{I})}=2\left(\frac{\mathbf{U}_{i}^{-1} \hat{\mathbf{v}}}{\left|\mathbf{U}_{i}^{-1} \hat{\mathbf{v}}\right|^{2}}-\mathbf{U}_{i} \hat{\mathbf{v}}\right), \quad \kappa^{(\mathrm{I})}=+1 \quad$ (type I solution $)$,

$\hat{\mathbf{n}}^{(\mathrm{II})}=\frac{2}{\rho}\left(\hat{\mathbf{v}}-\frac{\mathbf{U}_{i}^{2} \hat{\mathbf{v}}}{\left|\mathbf{U}_{i} \hat{\mathbf{v}}\right|^{2}}\right), \quad \mathbf{a}^{(\mathrm{II})}=\rho \mathbf{U}_{i} \hat{\mathbf{v}}, \quad \kappa^{(\mathrm{II})}=-1 \quad$ (type II solution $)$,

where $\rho$ is chosen such that $\left|\hat{\mathbf{n}}^{(\mathrm{II})}\right|=1$. $\mathbf{Q}^{(\mathrm{I})}$ and $\mathbf{Q}^{(\mathrm{II})}$ are obtained by substituting these back into equation (30). Moreover, the angles between the polarization on the two sides ( $\beta^{(\mathrm{I})}$ between $\mathbf{p}_{i}$ and $\kappa^{(\mathrm{I})} \mathbf{Q}^{(\mathrm{I})} \mathbf{p}_{j}$ for the type I twins and $\beta^{(\mathrm{II})}$ between $\mathbf{p}_{i}$ and $\kappa^{(\mathrm{II})} \mathbf{Q}^{(\mathrm{II})} \mathbf{p}_{j}$ for the type II twins) are given by

$$
\cos \beta^{(\mathrm{I}, \mathrm{II})}=\kappa^{(\mathrm{I}, \mathrm{II})} \hat{\mathbf{p}}_{\mathrm{i}} \cdot\left[\left(\mathbf{U}_{\mathrm{i}}+\mathbf{a}^{(\mathrm{I}, \mathrm{II})} \otimes \hat{\mathbf{n}}^{(\mathrm{I}, \mathrm{II})}\right) \mathbf{U}_{\mathrm{j}}^{-1}\right] \hat{\mathbf{p}}_{\mathrm{j}},
$$

where $\hat{\mathbf{p}}_{i}=\left(1 /\left|\mathbf{p}_{i}\right|\right) \mathbf{p}_{i}$ and $\hat{\mathbf{p}}_{j}=\left(1 /\left|\mathbf{p}_{j}\right|\right) \mathbf{p}_{j}$. Thus, these are $\beta^{(\mathrm{I}, \mathrm{II})}$ domain walls. Finally, if

$$
\mathbf{U}_{i} \mathbf{p}_{i}=\lambda \mathbf{p}_{i}, \quad \mathbf{U}_{j} \mathbf{p}_{j}=\lambda \mathbf{p}_{j}
$$

for some common $\lambda \neq 0$, then 


$$
\cos \beta^{(\mathrm{I}, \mathrm{II})}=\kappa^{(\mathrm{I}, \mathrm{II})} \hat{\mathbf{p}}_{i} \cdot \hat{\mathbf{p}}_{j}+\frac{1}{\lambda}\left(\mathbf{a}^{(\mathrm{I}, \mathrm{II})} \cdot \hat{\mathbf{p}}_{i}\right)\left(\hat{\mathbf{n}}^{(\mathrm{I}, \mathrm{II})} \cdot \hat{\mathbf{p}}_{i}\right) .
$$

Proof: The case $i=j$ is obvious. If $i \neq j$ and if equation (32) holds, we can verify that $\left(\mathbf{a}^{(\mathrm{I})}, \hat{\mathbf{n}}^{(\mathrm{I})}\right)$ and $\left(\mathbf{a}^{(\mathrm{II})}, \hat{\mathbf{n}}^{(\mathrm{II})}\right)$ in equations (33) and (34) respectively solve equations (30) (Bhattacharya 1992). We now show that $\left(\hat{\mathbf{n}}^{(\mathrm{I})}, \kappa^{(\mathrm{I})}\right)$ and $\left(\hat{\mathbf{n}}^{(\mathrm{II})}, \kappa^{(\mathrm{II})}\right)$ satisfy equation (31). According to equations (33) and (34),

$$
\mathbf{R}^{(\mathrm{I})} \hat{\mathbf{n}}^{(\mathrm{I})}=\hat{\mathbf{n}}^{(\mathrm{I})}, \quad \mathbf{R}^{(\mathrm{II})} \hat{\mathbf{n}}^{(\mathrm{II})}=-\hat{\mathbf{n}}^{(\mathrm{II})},
$$

so that, with an appropriate choice of $\kappa$,

$$
\left(\kappa \mathbf{R}^{\mathrm{T}} \mathbf{U}_{i}^{-1} \mathbf{p}_{i}-\mathbf{U}_{i}^{-1} \mathbf{p}_{i}\right) \cdot \hat{\mathbf{n}}=\kappa \mathbf{U}_{i}^{-1} \mathbf{p}_{i} \cdot \mathbf{R} \hat{\mathbf{n}}-\mathbf{U}_{i}^{-1} \mathbf{p}_{i} \cdot \hat{\mathbf{n}}=0 .
$$

Further, one can verify that

$$
\mathbf{U}_{i}^{-1} \mathbf{a}^{(\mathrm{I})} \cdot \mathbf{n}^{(\mathrm{I})}=\mathbf{U}_{i}^{-1} \mathbf{a}^{(\mathrm{II})} \cdot \mathbf{n}^{(\mathrm{II})}=0 .
$$

Now from equatons (32) and (39),

$$
\begin{aligned}
\left(\kappa \mathbf{R}^{\mathrm{T}} \mathbf{U}_{i}^{-1} \mathbf{p}_{i}-\mathbf{U}_{i}^{-1} \mathbf{p}_{i}\right) \cdot \hat{\mathbf{n}}=0 & \Leftrightarrow\left(\kappa \mathbf{R}^{T} \mathbf{U}_{i}^{-1} \mathbf{R} \mathbf{R}^{\mathrm{T}} \mathbf{p}_{i}-\mathbf{U}_{i}^{-1} \mathbf{p}_{i}\right) \cdot \hat{\mathbf{n}}=0 \\
& \Leftrightarrow\left(\kappa \mathbf{U}_{j}^{-1} \mathbf{p}_{j}-\mathbf{U}_{i}^{-1} \mathbf{p}_{i}\right) \cdot \hat{\mathbf{n}}=0 \\
& \Leftrightarrow\left(\kappa\left(\mathbf{Q} \mathbf{U}_{j}\right)^{-1} \mathbf{Q} \mathbf{p}_{j}-\mathbf{U}_{i}^{-1} \mathbf{p}_{i}\right) \cdot \hat{\mathbf{n}}=0 \\
& \Leftrightarrow\left(\kappa \mathbf{Q} \mathbf{p}_{j}-\mathbf{p}_{i}\right) \cdot \mathbf{U}_{i}^{-1} \hat{\mathbf{n}}=0 .
\end{aligned}
$$

The last identity follows since $\left(\mathbf{Q} \mathbf{U}_{j}\right)^{-\mathrm{T}} \hat{\mathbf{n}}=\mathbf{U}_{i}^{-\mathrm{T}} \hat{\mathbf{n}}$ as a consequence of equations (30) and (40). This gives equation (31). It also follows from Ball and James (1987) that these are the only solutions.

Finally, the angles $\beta^{(\mathrm{I}, \mathrm{II})}$ between $\mathbf{p}_{i}$ and $\kappa^{(\mathrm{I}, \mathrm{II})} \mathbf{Q}^{(\mathrm{I}, \mathrm{II})} \mathbf{p}_{j}$ follow from

$$
\begin{aligned}
\cos \beta^{(\mathrm{I}, \mathrm{II})} & =\frac{\kappa^{(\mathrm{I}, \mathrm{II})} \mathbf{Q}^{(\mathrm{I}, \mathrm{II})} \mathbf{p}_{j} \cdot \mathbf{p}_{i}}{\left|\mathbf{Q}^{(\mathrm{I}, \mathrm{II})} \mathbf{p}_{j}\right|\left|\mathbf{p}_{i}\right|} \\
& =\frac{\kappa^{(\mathrm{I}, \mathrm{II})}}{\left|\mathbf{p}_{i}\right|\left|\mathbf{p}_{j}\right|} \mathbf{p}_{i} \cdot\left[\left(\mathbf{U}_{i}+\mathbf{a}^{(\mathrm{I}, \mathrm{II})} \otimes \hat{\mathbf{n}}^{(\mathrm{I}, \mathrm{II})}\right) \mathbf{U}_{j}^{-1}\right] \mathbf{p}_{j} \\
& =\kappa^{(\mathrm{I}, \mathrm{II})} \hat{\mathbf{p}}_{i} \cdot \hat{\mathbf{p}}_{j}+\frac{\kappa^{(\mathrm{I}, \mathrm{II})}}{\lambda}\left(\mathbf{a}^{(\mathrm{I}, \mathrm{II})} \cdot \hat{\mathbf{p}}_{i}\right)\left(\hat{\mathbf{n}}^{\mathrm{II}, \mathrm{II})} \cdot \hat{\mathbf{p}}_{j}\right) \\
& =\kappa^{(\mathrm{I}, \mathrm{II})} \hat{\mathbf{p}}_{i} \cdot \hat{\mathbf{p}}_{j}+\frac{\kappa^{(\mathrm{I}, \mathrm{II})}}{\lambda}\left(\mathbf{a}^{(\mathrm{I}, \mathrm{II})} \cdot \hat{\mathbf{p}}_{i}\right)\left(\hat{\mathbf{n}}^{(\mathrm{I}, \mathrm{II})} \cdot \mathbf{R}^{(\mathrm{I}, \mathrm{II}) \mathrm{T}} \hat{\mathbf{p}}_{i}\right) \\
& =\kappa^{(\mathrm{I}, \mathrm{II})} \hat{\mathbf{p}}_{i} \cdot \hat{\mathbf{p}}_{j}+\frac{1}{\lambda}\left(\mathbf{a}^{(\mathrm{I}, \mathrm{II})} \cdot \hat{\mathbf{p}}_{i}\right)\left(\hat{\mathbf{n}}^{(\mathrm{I}, \mathrm{II})} \cdot \hat{\mathbf{p}}_{i}\right)
\end{aligned}
$$

because of equation (38). This completes the proof.

We have inherited the terminology type I and type II above from the twinning literature. In type I solutions, the interface is a crystallographic plane (rational plane) while in type II solutions the direction of shear $\left(\mathbf{U}_{i}^{-1} \mathbf{a}\right)$ is a crystallographic direction (rational direction). If a solution has both rational interface and direction of shear, we call it a compound solution.

Note that this lemma only gives us sufficient conditions for the existence of solutions. In other words it identifies a set of conditions under which we can find 
a solution but does not exclude the possibility of finding other solutions even if these symmetry conditions are violated. In fact we shall see one such solution later when we study the tetragonal to rhombohedral transition.

\subsection{Domain walls in barium titanate}

We now use lemma 1 to study possible domain walls in $\mathrm{BaTiO}_{3}$ for tetragonal, orthorhombic and rhombohedral phases.

\subsubsection{Tetragonal phase}

The energy wells $\mathcal{W}^{t}$ of the tetragonal phase are described in equation (17) and

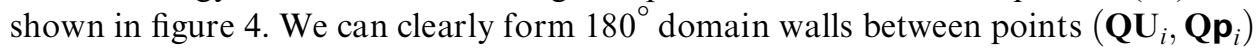
and $\left(\mathbf{Q U}_{i},-\mathbf{Q} \mathbf{p}_{i}\right)$ for any $\mathbf{Q} \in \mathbf{S O}(3)$ and $i=1,2,3$ according to lemma 1 .

We now turn to domain walls with different strains on the two sides. First consider the case $i=1$ and $j=2$. Note that $\mathbf{R}$, a twofold rotation about the axis $\hat{\mathbf{v}}=\left(1 / 2^{1 / 2}\right)\left(\hat{\mathbf{e}}_{1}+\hat{\mathbf{e}}_{2}\right)$ satisfies $\mathbf{R} \in \mathcal{P}_{0}$ (see appendix A) and equation (32). We can then conclude from lemma 1 that there are two possible solutions to equations (30) and (31) and they are obtained from equations (33) and (34) to be

$$
\begin{array}{cc}
\hat{\mathbf{n}}^{(\mathrm{I})}=\frac{1}{2^{1 / 2}}\left(\hat{\mathbf{e}}_{1}+\hat{\mathbf{e}}_{2}\right), & \mathbf{a}^{(\mathrm{I})}=\frac{2^{1 / 2}\left(\eta_{2}^{\mathrm{t}^{2}}-\eta_{1}^{\mathrm{t}^{2}}\right)}{\eta_{1}^{t^{2}}+\eta_{2}^{t^{2}}}\left(-\eta_{2}^{\mathrm{t}} \hat{\mathbf{e}}_{1}+\eta_{1}^{\mathrm{t}} \hat{\mathbf{e}}_{2}\right), \\
\hat{\mathbf{n}}^{(\mathrm{II})}=\frac{1}{2^{1 / 2}}\left(-\hat{\mathbf{e}}_{1}+\hat{\mathbf{e}}_{2}\right), & \mathbf{a}^{(\mathrm{II})}=\frac{2^{1 / 2}\left(\eta_{2}^{\mathrm{t}^{2}}-\eta_{1}^{\mathrm{t}^{2}}\right)}{\eta_{1}^{\mathrm{t}^{2}}+\eta_{2}^{t^{2}}}\left(\eta_{2}^{\mathrm{t}} \hat{\mathbf{e}}_{1}+\eta_{1}^{\mathrm{t}} \hat{\mathbf{e}}_{2}\right) .
\end{array}
$$

To obtain the angles $\beta^{(\mathrm{I}, \mathrm{II})}$ between the polarizations on the two sides, note that $\mathbf{U}_{1} \mathbf{p}_{1}=\eta_{2}^{\mathrm{t}} \mathbf{p}_{1}$ and $\mathbf{U}_{2} \mathbf{p}_{2}=\eta_{2}^{\mathrm{t}} \mathbf{p}_{2}$. Thus, we conclude from equation (37) that

$$
\cos \beta^{(\mathrm{I}, \mathrm{II})}=\frac{\eta_{1}^{\mathrm{t}^{2}}-\eta_{2}^{\mathrm{t}^{2}}}{\eta_{1}^{\mathrm{t}^{2}}+\eta_{2}^{\mathrm{t}^{2}}},
$$

whence from a standard trigonometric identity $\left(\tan (2 \beta)=(2 \tan \beta) /\left(1-\tan ^{2} \beta\right)\right)$

$$
\beta^{(\mathrm{I}, \mathrm{II})}=2 \tan ^{-1}\left(\frac{\eta_{2}^{t}}{\eta_{1}^{t}}\right) .
$$

For $\mathrm{BaTiO}_{3}, \beta^{(\mathrm{I}, \mathrm{II})}=90.62^{\circ}$. Thus, both solutions characterize $90^{\circ}$ domain walls.

To characterize these walls further, note that we could have obtained the same two solutions by choosing $\mathbf{R}$ to be a twofold rotation about $\hat{\mathbf{v}}^{*}=\left(1 / 2^{1 / 2}\right)\left(-\hat{\mathbf{e}}_{1}+\hat{\mathbf{e}}_{2}\right)$. Moreover, one can verify that the type I solution in terms of the rotation with axis $\hat{\mathbf{v}}$ coincides with the type II solution in terms of the rotation with axis $\hat{\mathbf{v}}^{*}$, and vice versa. As a result, these solutions are compound with a rational interface $\{110\}_{c}$ and a rational shear direction $\langle 110\rangle$.

The cases $i=1, j=3$ and $i=2, j=3$ similarly yield $\{110\}_{c}$ domain walls.

Thus, the domain walls in tetragonal wells can be characterized as shown in figure 4. Any point on one of energy wells can form a $180^{\circ}$ domain wall with a corresponding point on the well with the same strain but opposite polarization, and a $90^{\circ}$ domain wall with a point on each of the other wells. Further it is possible to show that these are the only domain walls that are possible in the tetragonal phase. This is in agreement with experimental observations (Hooton and Merz 1955, Jaffe et al. 1971, Xu 1991, Chou and Wayman 1997, Park and Chung 1997). 


\subsubsection{Orthorhombic phase}

The energy wells $\mathcal{W}^{\circ}$ of the orthorhombic phase are described in equation (19) and shown in figure 5. We can clearly form $180^{\circ}$ domain walls between points $\left(\mathbf{Q} \mathbf{U}_{i}, \mathbf{Q} \mathbf{p}_{i}\right)$ and $\left(\mathbf{Q \mathbf { U } _ { i }},-\mathbf{Q} \mathbf{p}_{i}\right)$ for any $\mathbf{Q} \in \mathbf{S O}(3)$ and $i=1, \ldots, 6$ according to lemma 1.

To find the other domain walls, let us begin with a pair $i, j$ such that $\mathbf{U}_{i}$ and $\mathbf{U}_{j}$ have the same $c$ axis, that is the pair 1 and 2, the pair 3 and 4 and the pair 5 and 6 . We shall first show that it is possible to form $90^{\circ}$ domain walls with normal $\{100\}_{c}$ between any of these pairs. Further these domain walls are obtained from compound solutions. Indeed, consider $i=1$ and $j=2$. Let $\mathbf{R}$ be a twofold rotation about the axis $\hat{\mathbf{v}}=\hat{\mathbf{e}}_{2}$. Note that $\mathbf{R} \in \mathcal{P}^{0}$ and satisfies equation (32). So we can obtain two solutions from equations (33) and (34), and they have normal $\hat{\mathbf{n}}^{(\mathrm{I})}=\hat{\mathbf{e}}_{2}$ and $\hat{\mathbf{n}}^{\text {(II) }}=\hat{\mathbf{e}}_{1}$. Further, we can calculate the angle between the polarizations by noting that $\mathbf{U}_{1} \mathbf{p}_{1}=\eta_{1}^{\mathrm{o}} \mathbf{p}_{1}$ and $\mathbf{U}_{2} \mathbf{p}_{2}=\eta_{1}^{\mathrm{o}} \mathbf{p}_{2}$ :

$$
\cos \beta^{(\mathrm{I}, \mathrm{II})}=\frac{-\eta_{1}^{\mathrm{o}^{2}}+\eta_{2}^{\mathrm{o}^{2}}}{\eta_{1}^{\mathrm{o}^{2}}+\eta_{2}^{\mathrm{o}^{2}}}
$$

where $\eta_{1}^{\mathrm{o}}=\frac{1}{2}\left(\alpha^{\mathrm{o}}+\delta^{\mathrm{o}}\right), \eta_{2}^{\mathrm{o}}=\frac{1}{2}\left(\alpha^{\mathrm{o}}-\delta^{\mathrm{o}}\right), \eta_{3}^{\mathrm{o}}=\beta^{\mathrm{o}}$ and $\alpha^{\mathrm{o}}, \delta^{\mathrm{o}}$, and $\beta^{\mathrm{o}}$ are lattice parameters of orthorhombic variants (20). For $\mathrm{BaTiO}_{3}, \beta^{(\mathrm{I}, \mathrm{II})}=90.13^{\circ}$. Finally, note that we obtain the same solutions by starting with a twofold rotation $\mathbf{R}$ about the axis $\hat{\mathbf{v}}=\hat{\mathbf{e}}_{2}$. The pair 3 and 4 and the pair 5 and 6 are treated analogously.

Now consider the remaining cases, that is when $\mathbf{U}_{i}$ and $\mathbf{U}_{j}$ do not have the same $c$ axis. We shall show that here we can form a type I $120^{\circ}$ domain wall with normal $\{110\}_{c}$ and a type II $60^{\circ}$ domain wall with an irrational normal. To be specific, consider the pairs $\left(\mathbf{U}_{1}, \pm \mathbf{p}_{1}\right)$ and $\left(\mathbf{U}_{4}, \pm \mathbf{p}_{4}\right)$. Let $\mathbf{R}$ be a twofold rotation about the axis $\hat{\mathbf{v}}=\left(1 / 2^{1 / 2}\right)\left(\hat{\mathbf{e}}_{2}+\hat{\mathbf{e}}_{3}\right)$. Note that $\mathbf{R} \in \mathcal{P}^{0}$ and satisfies equation (32). So we can obtain two solutions from equations (33) and (34). Further, one can verify that $\mathbf{U}_{1} \mathbf{p}_{1}=\eta_{1}^{\mathrm{o}} \mathbf{p}_{1}$ and $\mathbf{U}_{4} \mathbf{p}_{4}=\eta_{1}^{\mathrm{o}} \mathbf{p}_{4}$; so that the angles $\beta^{(\mathrm{I}, \mathrm{II})}$ between the polarizations are

$$
\begin{aligned}
& \cos \beta^{(\mathrm{I})}=\frac{\eta_{2}^{\mathrm{o}^{2}} \eta_{3}^{\mathrm{o}^{2}}-\eta_{1}^{\mathrm{o}^{2}}\left(2 \eta_{2}^{\mathrm{o}^{2}}+\eta_{3}^{\mathrm{o}^{2}}\right)}{\eta_{2}^{\mathrm{o}^{2}} \eta_{3}^{\mathrm{o}^{2}}+\eta_{1}^{\mathrm{o}^{2}}\left(2 \eta_{2}^{\mathrm{o}^{2}}+\eta_{3}^{\mathrm{o}^{2}}\right)}, \\
& \cos \beta^{(\mathrm{II})}=\frac{-\eta_{1}^{\mathrm{o}^{2}}+\eta_{2}^{\mathrm{o}^{2}}+2 \eta_{3}^{\mathrm{o}^{2}}}{\eta_{1}^{\mathrm{o}^{2}}+\eta_{2}^{\mathrm{o}^{2}}+2 \eta_{3}^{\mathrm{o}^{2}}} .
\end{aligned}
$$

For $\mathrm{BaTiO}_{3}, \beta^{(\mathrm{I})}=120.27^{\circ}$ and $\beta^{(\mathrm{II})}=60.27^{\circ}$. The remaining pairs of variants are treated analogously.

In summary, each point on any orthorhombic well can form a $180^{\circ}$ domain wall with the corresponding point on the well with the same strain but opposite polarization, a $90^{\circ}$ domain wall with one point each on the two wells with different strains with identical $c$ axis, and a $60^{\circ}$ or $120^{\circ}$ domain wall with one point on each well of the pair with different $c$ axes. Further it is possible to show that these are the only domain walls that are possible in the orthorhombic phase. This is shown schematically in figure 5 and is in agreement with observations (Jona and Shirane 1962, Huang et al. 1995). 


\subsubsection{Rhombohedral phase}

The energy wells $\mathcal{W}^{r}$ of the rhombohedral phase are described in equation (21) and shown in figure 6 . We can clearly form $180^{\circ}$ domain walls between points $\left(\mathbf{Q U _ { i } ^ { \mathrm { r } }}, \mathbf{Q} \mathbf{p}_{i}^{\mathrm{r}}\right)$ and $\left(\mathbf{Q \mathbf { U } _ { i } ^ { \mathrm { r } }},-\mathbf{Q} \mathbf{p}_{i}^{\mathrm{r}}\right)$ for any $\mathbf{Q} \in \mathrm{SO}(3)$ and $i=1, \ldots, 4$ according to lemma 1.

We now turn to domain walls with different strains on the two sides. First consider the case $i=1$ and $j=2$. Note that $\mathbf{R}$, a twofold rotation about the axis $\hat{\mathbf{v}}=\left(1 / 2^{1 / 2}\right)\left(\hat{\mathbf{e}}_{2}+\hat{\mathbf{e}}_{3}\right)$ satisfies $\mathbf{R} \in \mathcal{P}_{0} \quad$ (see appendix $\quad$ A) and $\mathbf{U}_{2}^{\mathrm{r}}=\mathbf{R}^{\mathrm{T}} \mathbf{U}_{1}^{\mathrm{r}} \mathbf{R}$, $\mathbf{p}_{2}^{\mathrm{r}}=\mathbf{R}^{\mathrm{T}} \mathbf{p}_{1}^{\mathrm{r}}$. We can then conclude from lemma 1 that there are two possible solutions to equations (30) and (31) and they are obtained from equations (33) and (34) to be

$$
\begin{array}{ll}
\hat{\mathbf{n}}^{(\mathrm{I})}=\frac{1}{2^{1 / 2}}\left(\hat{\mathbf{e}}_{2}+\hat{\mathbf{e}}_{3}\right), & \mathbf{a}^{(\mathrm{I})}=\frac{2^{3 / 2} \delta^{\mathrm{r}}\left(2 \alpha^{\mathrm{r}}+\delta^{\mathrm{r}}\right)}{\alpha^{\mathrm{r}^{2}}+2 \delta^{\mathrm{r}^{2}}}\left\{-\alpha^{\mathrm{r}} \hat{\mathbf{e}}_{1}-\delta^{\mathrm{r}} \hat{\mathbf{e}}_{2}-\delta^{\mathrm{r}} \hat{\mathbf{e}}_{3}\right\}, \\
\hat{\mathbf{n}}^{(\mathrm{II})}=-\hat{\mathbf{e}}_{1}, & \mathbf{a}^{(\mathrm{II})}=\frac{2 \delta^{r}\left(2 \alpha^{\mathrm{r}}+\delta^{\mathrm{r}}\right)}{\alpha^{\mathrm{r}^{2}}+2 \alpha^{\mathrm{r}} \delta^{\mathrm{r}}+3 \delta^{\mathrm{r}^{\mathrm{r}}}}\left\{2 \delta^{\mathrm{r}} \hat{\mathbf{e}}_{1}+\left(\alpha^{\mathrm{r}}+\delta^{\mathrm{r}}\right) \hat{\mathbf{e}}_{2}+\left(\alpha^{\mathrm{r}}+\delta^{\mathrm{r}}\right) \hat{\mathbf{e}}_{3}\right\} .
\end{array}
$$

To obtain the angles $\beta^{(\mathrm{I}, \mathrm{II})}$ between the polarizations on the two sides, first note that in general there does not exist the common $\lambda$ such that equation (36) holds unless the lattice parameters of rhombohedral variants have certain special relation. Thus, we have to resort to equation (35) with the help of equations (44). It follows that

$$
\begin{aligned}
& \cos \beta^{(\mathrm{I})}=\frac{\alpha^{\mathrm{r}^{2}}-8 \alpha^{\mathrm{r}} \delta^{\mathrm{r}}-2 \delta^{\mathrm{r}^{2}}}{3 \alpha^{\mathrm{r}^{2}}+6 \delta^{\mathrm{r}^{2}}}, \\
& \cos \beta^{(\mathrm{II})}=-\frac{\alpha^{\mathrm{r}^{2}}+10 \alpha^{\mathrm{r}} \delta^{\mathrm{r}}+7 \delta^{\mathrm{r}^{2}}}{3\left(\alpha^{\mathrm{r}^{2}}+2 \alpha^{\mathrm{r}} \delta^{\mathrm{r}}+3 \delta^{\mathrm{r}^{2}}\right)} .
\end{aligned}
$$

For $\mathrm{BaTiO}_{3}, \beta^{(\mathrm{I})}=70.74^{\circ}$ and $\beta^{(\mathrm{II})}=109.68^{\circ}$.

To characterize these walls further, note that we could have obtained the same two solutions by choosing $\mathbf{R}$ to be a twofold rotation about $\hat{\mathbf{v}}^{*}=\hat{\mathbf{e}}_{1}$. Moreover, one can verify that the type I solution in terms of the rotation with axis $\hat{\mathbf{v}}$ coincides with the type II solution in terms of the rotation with axis $\hat{\mathbf{v}}^{*}$, and vice versa. As a result, these solutions are compound.

The remaining cases can be treated analogously.

In summary, the domain walls in rhombohedral wells can be characterized as shown in figure 6. Any point on one of energy wells can form a $180^{\circ}$ domain wall with a corresponding point on the well with the same strain but opposite polarization, and a $70^{\circ}$ or $109^{\circ}$ domain wall with one point on each of the other wells with different strains. Further it is possible to show that these are the only domain walls that are possible in the rhombohedral phase. This is in general agreement with experimental observations of the rhombohedral domain configuration (Jona and Shirane 1962, Ricote et al. 2000).

\subsection{Rhomobohedral-Tetragonal domain wall}

We have discussed in $\S 2.3$ that PZT at compositions at the morphotropic phase boundary can be polarized in either the $\langle 100\rangle$ or the $\langle 111\rangle$ directions, and the energy 
has both the tetragonal and the rhombohedral wells. This material, therefore, can switch from the rhombohedral to the tetragonal phase and vice versa. Further, the rhombohedral-to-tetragona 1 phase transformation in relaxors is also the basis of the ultrahigh strains (Shrout and Park 1997). A similar transformation has recently also been observed in $\mathrm{BaTiO}_{3}$ (Park et al. 1999).

We now explore whether it is possible to form a low-energy interface between with the $\langle 100\rangle$ polarized tetragonal one side and the $\langle 111\rangle$ polarized rhombohedral on the other. This would require us to satisfy the mechanical and electrical compatibility conditions (30) and (31) with $\mathbf{U}_{j}=\mathbf{U}_{1}^{\mathrm{r}}, \mathbf{U}_{i}=\mathbf{U}_{1}^{\mathrm{t}}, \mathbf{p}_{j}=\mathbf{p}_{1}^{\mathrm{r}}, \mathbf{p}_{i}=\mathbf{p}_{1}^{\mathrm{t}}$. Ball and James (1987) have given the most general conditions under which one can solve equation (30). According to their result, a solution is possible if and only if the eigenvalues $\lambda_{1}, \lambda_{2}$ and $\lambda_{3}$ of the matrix $\left(\mathbf{U}_{1}^{\mathrm{t}}\right)^{-1}\left(\mathbf{U}_{1}^{\mathrm{r}}\right)^{2}\left(\mathbf{U}_{1}^{\mathrm{t}}\right)^{-1}$ satisfies $0 \leqslant \lambda_{1} \leqslant \lambda_{2}=1 \leqslant \lambda_{3}$. If these conditions are satisfied, then their result also provides us with a formula for $\hat{\mathbf{n}}$. Using this value of $\hat{\mathbf{n}}$ we can look for solutions of equation (31). The calculations, which are omitted since they are tedious and unenlightening, lead us to conclude the following.

It is possible to form a low-energy domain wall between the rhombohedral and tetragonal phases if and only if

$$
\begin{aligned}
\eta-\xi & =\eta_{1}^{2}, \\
\eta(\eta+\xi)+\eta_{1}^{2} \eta_{2}^{2} & \leqslant \eta_{1}^{2} \eta+\eta_{2}^{2}(\eta+\xi)+2 \xi^{2}, \\
\xi^{r}\left[2 \eta_{1}+\lambda \epsilon_{1}+(1-\lambda) \epsilon_{3}\right] & =\kappa\left[\lambda \epsilon_{1}+(1-\lambda) \epsilon_{3}\right]\left(\alpha^{\mathrm{r}}+2 \delta^{\mathrm{r}}\right) \xi^{\mathrm{t}},
\end{aligned}
$$

where

$$
\begin{gathered}
\lambda=\frac{1}{1-\hat{\kappa}\left\{\left[\left(\lambda_{3}-1\right) /\left(1-\lambda_{1}\right)\right]\left[\left(2+\epsilon_{1}^{2}\right) /\left(2+\epsilon_{3}^{2}\right)\right]\right\}^{1 / 2}}, \\
\lambda_{1}=\frac{1}{2 \eta_{1}^{2} \eta_{2}^{2}}\left(\eta_{1}^{2} \eta+\eta_{2}^{2}(\eta+\xi)-\left\{\left[\eta_{1}^{2} \eta-\eta_{2}^{2}(\eta+\xi)\right]^{2}+8 \eta_{1}^{2} \eta_{2}^{2} \xi^{2}\right\}^{1 / 2}\right), \\
\lambda_{3}=\frac{1}{2 \eta_{1}^{2} \eta_{2}^{2}}\left(\eta_{1}^{2} \eta+\eta_{2}^{2}(\eta+\xi)+\left\{\left[\eta_{1}^{2} \eta-\eta_{2}^{2}(\eta+\xi)\right]^{2}+8 \eta_{1}^{2} \eta_{2}^{2} \xi^{2}\right\}^{1 / 2}\right), \\
\epsilon_{1}=\frac{1}{2 \eta_{1} \eta_{2} \xi}\left(\eta_{1}^{2} \eta-\eta_{2}^{2}(\eta+\xi)-\left\{\left[\eta_{1}^{2} \eta-\eta_{2}^{2}(\eta+\xi)\right]^{2}+8 \eta_{1}^{2} \eta_{2}^{2} \xi^{2}\right\}^{1 / 2}\right), \\
\epsilon_{3}=\frac{1}{2 \eta_{1} \eta_{2} \xi}\left(\eta_{1}^{2} \eta-\eta_{2}^{2}(\eta+\xi)+\left\{\left[\eta_{1}^{2} \eta-\eta_{2}^{2}(\eta+\xi)\right]^{2}+8 \eta_{1}^{2} \eta_{2}^{2} \xi^{2}\right\}^{1 / 2}\right), \\
\eta_{1}=\eta_{1}^{\mathrm{t}}, \quad \eta_{2}=\eta_{2}^{\mathrm{t}}, \quad \eta=\left(\alpha^{\mathrm{r}}\right)^{2}+2\left(\delta^{\mathrm{r}}\right)^{2}, \quad \xi=\delta^{\mathrm{r}}\left(2 \alpha^{\mathrm{r}}+\delta^{\mathrm{r}}\right), \kappa= \pm 1, \hat{\kappa}= \pm 1 .
\end{gathered}
$$

Note that these are severe restrictions on $\eta_{1}^{\mathrm{t}}, \eta_{2}^{\mathrm{t}}, \alpha^{\mathrm{r}}, \delta^{\mathrm{r}}, \xi^{\mathrm{t}}, \xi^{\mathrm{r}}$ which are given for any given material. We conclude therefore that only very special materials can form these low-energy rhombohedral-tetragonal walls. We speculate that materials whose parameters satisfy these special relations can undergo the rhombohedral-to-tetrago nal phase transformation under much smaller driving forces, and thus with much smaller hysteresis, than the others. We wonder whether these relations have any connection to the relaxors that display ultrahigh strains. We note here that such special relations play a very significant role in the context of martensites where it has 
been established that special relations can lead to very special microstructures (Bhattacharya 1991).

\subsection{Other domain patterns}

\subsubsection{Banded domain patterns}

We now use the same ideas to examine the more complex domain patterns that are observed in ferroelectric materials. Consider a state shown in figure $8(a)$ where $\left(\mathbf{F}^{+}, \mathbf{p}^{+}\right)$and $\left(\mathbf{F}^{-}, \mathbf{p}^{-}\right)$alternate in layers separated by parallel planes with normal $\hat{\mathbf{n}}$ in the reference configuration. Notice that the necessary conditions that this layered structure be an energy minimizer are the same as those for forming a single domain

(a)
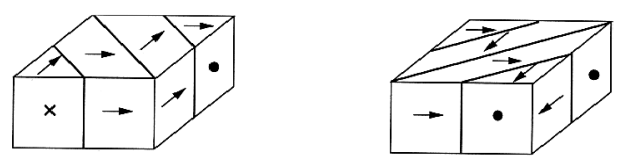

(b)

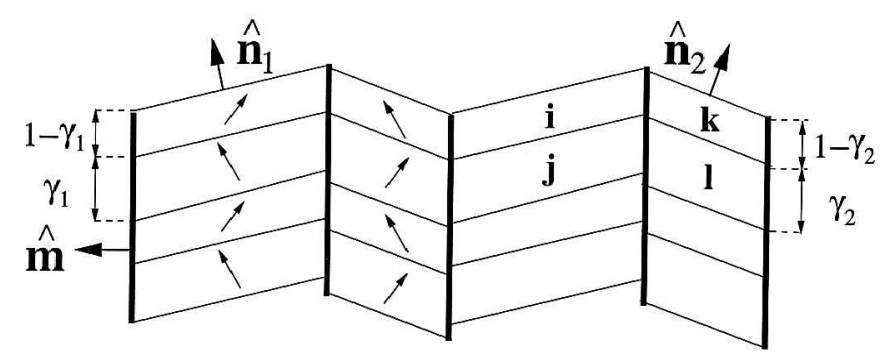

(c)
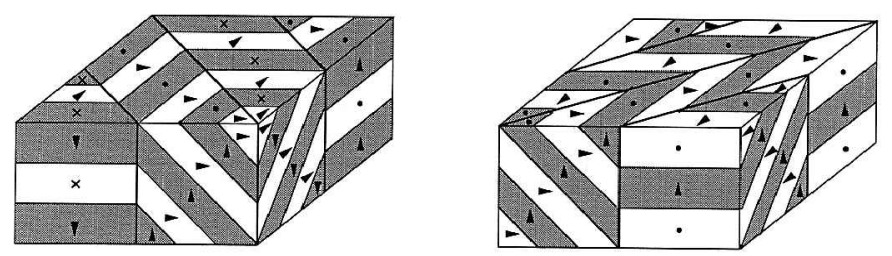

(d)

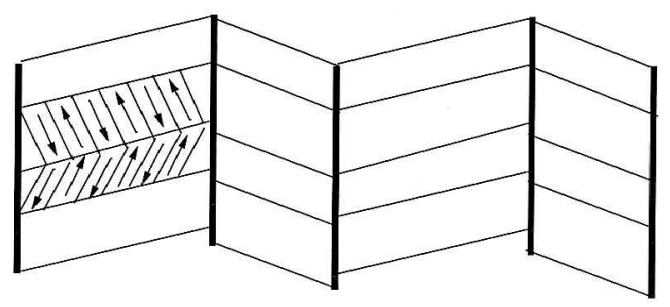

Figure 8. (a) Rank-one lamellar domain patterns. (b) Rank-two banded domain patterns. (c) Predicted banded domain patterns for ferroelectric crystals in the tetragonal phase. (d) High-rank domain patterns with very-fine-scale electric substructure within sublayers. 
wall. Therefore, the results above describe not only a single domain wall but also a laminate made of many parallel domain walls.

Let us now turn to the more complicated banded structure shown schematically in figure $8(b)$. This is commonly observed (for example Jona and Shirane (1962) and Arlt (1990)) and consists of parallel 'layers' which themselves are made up of many parallel 'sublayers'. The length scale of the sublayers is typically much smaller than those of the layers. For this structure to be an energy minimizer, the four states must belong to energy wells; let us label the corresponding wells $i, j, k$ and $l$. The sublayers must satisfy both the mechanical and electrical compatibility conditions (30) and (31) across the interfaces with normals $\hat{\mathbf{n}}_{1}$ and $\hat{\mathbf{n}}_{2}$ as before. Finally the layers themselves must satisfy the mechanical and electrical compatibility conditions across the interfaces with normal $\hat{\mathbf{m}}$. However, this compatibility is only on average (James and Kinderlehrer 1993). Since the sublayers are much finer than the layers, at the level of the layers we do not see the individual sublayers; instead we find the average deformation gradient and the average polarization and only require these averages to be compatible across the interface with normal $\hat{\mathbf{m}}$. Putting all this together, we conclude the following.

We can form a structure shown in figure $8(b)$ as an energy minimizer if, for given $i, j, k, l$, we satisfy the equations

$$
\begin{aligned}
& \mathbf{Q}_{1} \mathbf{U}_{j}-\mathbf{U}_{i}=\mathbf{a}_{1} \otimes \hat{\mathbf{n}}_{1}, \quad\left(\kappa_{1} \mathbf{Q}_{1} \mathbf{p}_{j}-\mathbf{p}_{i}\right) \cdot \mathbf{U}_{i}^{-\mathrm{T}} \hat{\mathbf{n}}_{1}=0, \\
& \mathbf{Q}_{2} \mathbf{U}_{l}-\mathbf{U}_{k}=\mathbf{a}_{2} \otimes \hat{\mathbf{n}}_{2}, \quad\left(\kappa_{2} \mathbf{Q}_{2} \mathbf{p}_{l}-\mathbf{p}_{k}\right) \cdot \mathbf{U}_{k}^{-\mathrm{T}} \hat{\mathbf{n}}_{2}=0, \\
& \mathbf{Q} \tilde{\mathbf{F}}-\tilde{\mathbf{G}}=\mathbf{b} \otimes \hat{\mathbf{m}}, \quad(\kappa \mathbf{Q} \tilde{\mathbf{p}}-\tilde{\mathbf{q}}) \cdot \tilde{\mathbf{G}}^{-\mathrm{T}} \hat{\mathbf{m}}=0,
\end{aligned}
$$

where

$$
\begin{aligned}
& \tilde{\mathbf{F}}=\gamma_{2} \mathbf{Q}_{2} \mathbf{U}_{l}+\left(1-\gamma_{2}\right) \mathbf{U}_{k}=\mathbf{U}_{k}+\gamma_{2} \mathbf{a}_{2} \otimes \hat{\mathbf{n}}_{2}, \\
& \tilde{\mathbf{G}}=\gamma_{1} \mathbf{Q}_{1} \mathbf{U}_{j}+\left(1-\gamma_{1}\right) \mathbf{U}_{i}=\mathbf{U}_{i}+\gamma_{1} \mathbf{a}_{1} \otimes \hat{\mathbf{n}}_{1}, \\
& \tilde{\mathbf{p}}=\gamma_{2} \kappa_{2} \mathbf{Q}_{2} \mathbf{p}_{l}+\left(1-\gamma_{2}\right) \mathbf{p}_{k}, \\
& \tilde{\mathbf{q}}=\gamma_{1} \kappa_{1} \mathbf{Q}_{1} \mathbf{p}_{j}+\left(1-\gamma_{1}\right) \mathbf{p}_{i}
\end{aligned}
$$

for some vectors $\mathbf{a}_{1}, \mathbf{a}_{2}, \mathbf{b}, \hat{\mathbf{n}}_{1}, \hat{\mathbf{n}}_{2}$ and $\hat{\mathbf{m}}$, for rotations $\mathbf{Q}_{1}, \mathbf{Q}_{2}$ and $\mathbf{Q}$ and for $\kappa_{1}, \kappa_{2}, \kappa= \pm 1$.

Note that equations (50) and (51) are the same as equations (30) and (31) considered earlier and can be solved using lemma 1. It turns out that it is rather difficult to solve equation (52) in general; we can find solutions to the first equation here, but the latter makes it over-determined. There are, however, some very interesting solutions that one can obtain using symmetry for the case when we have equal volume fractions $\gamma_{1}=\gamma_{2}=\gamma$. Assume that the pair $\left(\mathbf{U}_{i}, \mathbf{p}_{i}\right)$ and $\left(\mathbf{U}_{j}, \mathbf{p}_{j}\right)$ and the pair $\left(\mathbf{U}_{k}, \mathbf{p}_{k}\right)$ and $\left(\mathbf{U}_{l}, \mathbf{p}_{l}\right)$ satisfy the conditions listed in lemma 1 . Then one can obtain solutions to equations (50) and (51). Further, suppose that we can find a twofold rotation $\mathbf{R}$ about the axis $\hat{\mathbf{v}}$ that relates the variants as follows:

$$
\begin{aligned}
& \mathbf{U}_{l}=\mathbf{R} \mathbf{U}_{j} \mathbf{R}, \quad \mathbf{p}_{l}=\kappa_{1} \kappa_{2} \mathbf{R} \mathbf{p}_{j}, \\
& \mathbf{U}_{k}=\mathbf{R} \mathbf{U}_{i} \mathbf{R}, \quad \mathbf{p}_{k}=\mathbf{R} \mathbf{p}_{i} \text {. }
\end{aligned}
$$


It follows that

$$
\mathbf{Q}_{2}=\mathbf{R} \mathbf{Q}_{1} \mathbf{R}, \quad \mathbf{a}_{2}=\mathbf{R} \mathbf{a}_{1}, \quad \hat{\mathbf{n}}_{2}=\mathbf{R} \hat{\mathbf{n}}_{1} .
$$

Therefore, with $\gamma_{1}=\gamma_{2}=\gamma$,

$$
\tilde{\mathbf{F}}=\mathbf{R} \tilde{\mathbf{G}} \mathbf{R}, \quad \tilde{\mathbf{p}}=\mathbf{R} \tilde{\mathbf{q}} .
$$

Now a slight modification of case 2 in lemma 1 shows that there are two solutions to equation (52) given by

$$
\begin{gathered}
\hat{\mathbf{m}}^{(\mathrm{I})}=\hat{\mathbf{v}}, \quad \mathbf{b}^{(\mathrm{I})}=2\left(\frac{\tilde{\mathbf{G}}^{-\mathrm{T}} \hat{\mathbf{v}}}{\left|\tilde{\mathbf{G}}^{-\mathrm{T}} \hat{\mathbf{v}}\right|^{2}}-\tilde{\mathbf{G}} \hat{\mathbf{v}}\right), \quad \kappa^{(\mathrm{I})}=+1, \\
\hat{\mathbf{m}}^{(\mathrm{II})}=\frac{2}{\rho}\left(\hat{\mathbf{v}}-\frac{\tilde{\mathbf{G}}^{\mathrm{T}} \tilde{\mathbf{G}} \hat{\mathbf{v}}}{|\tilde{\mathbf{G}} \hat{\mathbf{v}}|^{2}}\right), \quad \mathbf{m}^{(\mathrm{II})}=\rho \mathbf{U}_{i} \hat{\mathbf{v}}, \quad \kappa^{(\mathrm{II})}=-1,
\end{gathered}
$$

where $\rho$ is chosen such that $\left|\hat{\mathbf{m}}^{(\mathrm{II})}\right|=1$.

Let us now specialize to tetragonal variants with energy wells (34). For the case $i=1, j=2, k=1, l=2$ involving only two variants, we can find solutions with normals

$$
\hat{\mathbf{m}}^{(\mathrm{I})}=\left(\begin{array}{l}
1 \\
0 \\
0
\end{array}\right), \quad \hat{\mathbf{m}}^{(\mathrm{II})}=\left(\begin{array}{l}
0 \\
1 \\
0
\end{array}\right) .
$$

For the case $i=3, j=2, k=3, l=1$, which involves all three variants as shown in figure $8(c)$, we also have two solutions

$$
\hat{\mathbf{m}}^{(\mathrm{I})}=\frac{1}{\sqrt{2}}\left(\begin{array}{c}
-1 \\
1 \\
0
\end{array}\right), \quad \hat{\mathbf{m}}^{(\mathrm{II})}=\frac{1}{2^{1 / 2}}\left(\begin{array}{c}
\left(\frac{1}{2 w^{2}+1}\right)^{1 / 2} \\
\left(\frac{1}{2 w^{2}+1}\right)^{1 / 2} \\
\left(\frac{4 w^{2}}{2 w^{2}+1}\right)^{1 / 2}
\end{array}\right),
$$

where

$$
w=\frac{(\gamma-1)\left(\eta_{1}^{t^{2}}-\eta_{2}^{t^{2}}\right)}{2 \eta_{1}^{t^{2}}-\left(\eta_{1}^{t^{2}}-\eta_{2}^{t^{2}}\right) \gamma}
$$

Notice that, if $\eta_{2}^{\mathrm{t}} / \eta_{1}^{\mathrm{t}} \approx 1$, then $\hat{\mathbf{m}}^{(\mathrm{II})} \approx\left(1 / 2^{1 / 2}\right)(1,1,0)^{\mathrm{T}}$. All other sets of variants are obtained from the above by symmetry.

Let us go back briefly to the case $\gamma_{1} \neq \gamma_{2}$. Here, as we have already noted, there are no generic solutions to equations (52). It is however, possible to form such domain patterns by simply introducing an 'electrical substructure' within the sublayer as shown in figure $8(d)$. Recall that, if $(\mathbf{F}, \mathbf{p}) \in \mathcal{W}$, then $(\mathbf{F},-\mathbf{p}) \in \mathcal{W}$. So within each sublayer we make a substructure of $180^{\circ}$ domains so that the average polarization is zero. We are then left with only the mechanical expressions in equations (50)(52), which have many solutions. 
Finally, we note that we can form an infinite number of such pattern s by iterating this construction and obtaining layers within layers within layers and so forth.

\subsubsection{Crossing domain pattern}

Consider the crossing pattern involving four different polarized states shown in figure 9. The key difference between this and the banded layered pattern shown in figure $8(b)$ is that here we do not have any separation of length scales, that is all the widths are comparable and all adjacent pairs form 'exact' interfaces. Therefore the material has to satisfy a much more restrictive condition to satisfy such a pattern to be an energy-minimizing state.

The deformation gradient and polarization associated with the different states must belong to the energy wells. So, without loss of generality, let us assume that they are given by $\left(\mathbf{U}_{i}, \mathbf{p}_{i}\right), \quad\left(\mathbf{Q}_{j} \mathbf{U}_{j}, \kappa_{j} \mathbf{Q}_{j} \mathbf{p}_{j}\right), \quad\left(\mathbf{Q}_{j} \mathbf{Q}_{k} \mathbf{U}_{k}, \kappa_{j} \kappa_{k} \mathbf{Q}_{j} \mathbf{Q}_{k} \mathbf{p}_{k}\right)$, $\left(\mathbf{Q}_{j} \mathbf{Q}_{k} \mathbf{Q}_{l} \mathbf{U}_{l}, \kappa_{j} \kappa_{k} \kappa_{l} \mathbf{Q}_{j} \mathbf{Q}_{k} \mathbf{Q}_{l} \mathbf{p}_{l}\right)$ for suitable choices of $i, j, k, l$ and where as usual $\mathbf{Q}_{j}, \mathbf{Q}_{k}, \mathbf{Q}_{l} \in \mathrm{SO}(3)$ and $\kappa_{i}, \kappa_{j}, \kappa_{k}, \kappa_{l}= \pm 1$. Further these states must satisfy mechanical and electrical compatibility conditions (30) and (31) across the interfaces:

$$
\begin{aligned}
& \mathbf{Q}_{j} \mathbf{U}_{j}-\mathbf{U}_{i}=\mathbf{a}_{1} \otimes \hat{\mathbf{n}}_{1}, \quad\left(\kappa_{j} \mathbf{Q}_{j} \mathbf{p}_{j}-\mathbf{p}_{i}\right) \cdot \mathbf{U}_{i}^{-\mathrm{T}} \hat{\mathbf{n}}_{i}=0, \\
& \mathbf{Q}_{k} \mathbf{U}_{k}-\mathbf{U}_{j}=\mathbf{a}_{2} \otimes \hat{\mathbf{n}}_{2}, \quad\left(\kappa_{k} \mathbf{Q}_{k} \mathbf{p}_{k}-\mathbf{p}_{j}\right) \cdot \mathbf{U}_{j}^{-\mathrm{T}} \hat{\mathbf{n}}_{j}=0, \\
& \mathbf{Q}_{l} \mathbf{U}_{l}-\mathbf{U}_{k}=\mathbf{a}_{3} \otimes \hat{\mathbf{n}}_{3}, \quad\left(\kappa_{l} \mathbf{Q}_{l} \mathbf{p}_{l}-\mathbf{p}_{k}\right) \cdot \mathbf{U}_{k}^{-\mathrm{T}} \hat{\mathbf{n}}_{k}=0, \\
& \mathbf{Q}_{i} \mathbf{U}_{i}-\mathbf{U}_{l}=\mathbf{a}_{4} \otimes \hat{\mathbf{n}}_{4}, \quad\left(\kappa_{i} \mathbf{Q}_{i} \mathbf{p}_{i}-\mathbf{p}_{l}\right) \cdot \mathbf{U}_{l}^{-\mathrm{T}} \hat{\mathbf{n}}_{l}=0, \\
& \mathbf{Q}_{i} \mathbf{Q}_{j} \mathbf{Q}_{k} \mathbf{Q}_{l}=\mathbf{I}, \quad \kappa_{i} \kappa_{j} \kappa_{k} \kappa_{l}=1,
\end{aligned}
$$

and $\hat{\mathbf{n}}_{1}, \ldots, \hat{\mathbf{n}}_{4}$ must lie on a plane to ensure that the four interfaces meet along a line. It is easy to verify that these conditions also prevent the formation of singularities at the fourfold corner, and are sufficient for forming the pattern shown in figure 9.

The crossing pattern was analysed in martensitic materials by Bhattacharya (1996), where one has to deal with only mechanical compatibility. In particular, he provided sufficient conditions for the expressions of mechanical compatibility
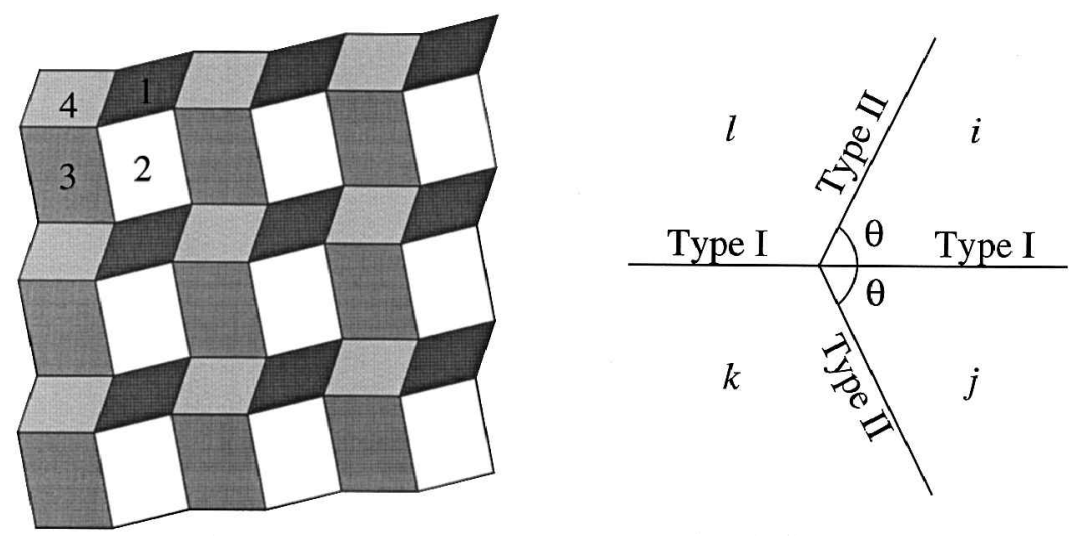

Figure 9. A crossing domain pattern consisting of four states. The structure predicted by lemma 2 : the $i-j$ and $k-l$ interfaces are of type I twins and lie on the same plane, while the $j-k$ and $l-i$ interfaces are of type II twins and are related by a reflection. 
in equations (62). It turns out, as in lemma 1, that these conditions are also sufficient to satisfy the electrical compatibility in equations (62) as well. The results are summarized in the following lemma which can be obtained by combining the arguments given by Bhattacharya (1996) with those in lemma 1.

Lemma 2: Let $\mathbf{U}_{i}, \mathbf{U}_{j}, \mathbf{U}_{k}, \mathbf{U}_{l}$ and $\mathbf{p}_{i}, \mathbf{p}_{j}, \mathbf{p}_{k}, \mathbf{p}_{l}$ be distinct (i.e., $i, j, k, l$ are distinct) and be related by twofold rotations $\mathbf{R}_{1}$ and $\mathbf{R}_{2}$ as follows:

$$
\begin{array}{r}
\mathbf{U}_{j}=\mathbf{R}_{1}^{\mathrm{T}} \mathbf{U}_{i} \mathbf{R}_{1}, \quad \mathbf{U}_{k}=\mathbf{R}_{2}^{\mathrm{T}} \mathbf{U}_{j} \mathbf{R}_{2}, \quad \mathbf{U}_{l}=\mathbf{R}_{1}^{\mathrm{T}} \mathbf{U}_{k} \mathbf{R}_{1}, \\
\mathbf{p}_{j}=\mathbf{R}_{1}^{\mathrm{T}} \mathbf{p}_{i}, \quad \mathbf{p}_{k}=\mathbf{R}_{2}^{\mathrm{T}} \mathbf{p}_{j}, \quad \mathbf{p}_{l}=\mathbf{R}_{1}^{\mathrm{T}} \mathbf{p}_{k}, \\
\mathbf{R}_{1}=-\mathbf{I}+2 \hat{\mathbf{v}}_{1} \otimes \hat{\mathbf{v}}_{1}, \quad \mathbf{R}_{2}=-\mathbf{I}+2 \hat{\mathbf{v}}_{2} \otimes \hat{\mathbf{v}}_{2},
\end{array}
$$

where $\hat{\mathbf{v}}_{1}$ and $\hat{\mathbf{v}}_{2}$ are the axes of rotations for $\mathbf{R}_{1}$ and $\mathbf{R}_{2}$ and satisfy

$$
\hat{\mathbf{v}}_{1} \cdot \hat{\mathbf{v}}_{2}=\mathbf{0}, \quad \hat{\mathbf{v}}_{3} \cdot \mathbf{U}_{i}^{2} \hat{\mathbf{v}}_{2} \neq \mathbf{0}
$$

with $\hat{\mathbf{v}}_{3}$ perpendicular to both $\hat{\mathbf{v}}_{1}$ and $\hat{\mathbf{v}}_{2}$. Then, the following solves equations (62):

$$
\begin{array}{lll}
\hat{\mathbf{n}}_{i}=\hat{\mathbf{v}}_{1}, & \mathbf{a}_{i}=2\left(\frac{\mathbf{U}_{i}^{-1} \hat{\mathbf{v}}_{1}}{\left|\mathbf{U}_{i}^{-1} \hat{\mathbf{v}}_{1}\right|^{2}}-\mathbf{U}_{i} \hat{\mathbf{v}}_{1}\right), & \kappa_{i}=-1, \\
\hat{\mathbf{n}}_{j}=\frac{2}{\rho}\left(\hat{\mathbf{v}}_{2}-\frac{\mathbf{U}_{j}^{2} \hat{\mathbf{v}}_{2}}{\left|\mathbf{U}_{j} \hat{\mathbf{v}}_{2}\right|^{2}}\right), & \mathbf{a}_{j}=\rho \mathbf{U}_{j} \hat{\mathbf{v}}_{2}, & \kappa_{j}=1, \\
\hat{\mathbf{n}}_{k}=\hat{\mathbf{v}}_{1}, & \mathbf{a}_{k}=2\left(\frac{\mathbf{U}_{k}^{-1} \hat{\mathbf{v}}_{1}}{\left|\mathbf{U}_{k}^{-1} \hat{\mathbf{v}}_{1}\right|^{2}}-\mathbf{U}_{k} \hat{\mathbf{v}}_{1}\right), & \kappa_{k}=-1, \\
\hat{\mathbf{n}}_{l}=\frac{2}{\rho^{\prime}}\left(\hat{\mathbf{v}}_{2}-\frac{\mathbf{U}_{l}^{2} \hat{\mathbf{v}}_{2}}{\left|\mathbf{U}_{l} \hat{\mathbf{v}}_{2}\right|^{2}}\right), & \mathbf{a}_{l}=\rho^{\prime} \mathbf{U}_{l} \hat{\mathbf{v}}_{2}, & \kappa_{l}=1,
\end{array}
$$

where $\rho$ and $\rho^{\prime}$ are chosen such that $\left|\hat{\mathbf{n}}_{j}\right|=\left|\hat{\mathbf{n}}_{l}\right|=1$.

Let us now study the consequences of this result. Note that, since the lemma requires all four states to be distinct, it gives no solution for the tetragonal phase. In fact, one can use the results of Bhattacharya (1996) to rule out all any crossing patterns in cubic-to-tetragonal transformations.

On the other hand, the lemma delivers one set of solutions for the rhombohedral phase. One solution corresponds to the choice

$$
i=1, \quad j=2, \quad k=3, \quad l=4,
$$

and those obtained from this by symmetry. For the specific choice (66), we can satisfy the hypothesis in the lemma by choosing $\hat{\mathbf{v}}_{1}=\hat{\mathbf{e}}_{1}$ and $\hat{\mathbf{v}}_{2}=\hat{\mathbf{e}}_{2}$; we obtain the normal in the reference configuration to be:

$$
\hat{\mathbf{n}}_{i}=\hat{\mathbf{e}}_{1}, \quad \hat{\mathbf{n}}_{j}=\frac{1}{2^{1 / 2}}\left(\hat{\mathbf{e}}_{1}-\hat{\mathbf{e}}_{3}\right), \quad \hat{\mathbf{n}}_{k}=\hat{\mathbf{e}}_{1}, \quad \hat{\mathbf{n}}_{l}=\frac{1}{2^{1 / 2}}\left(\hat{\mathbf{e}}_{1}+\hat{\mathbf{e}}_{3}\right) .
$$

In the orthorhombic phase, however, the lemma delivers two sets of solutions. One solution corresponds to the choice

$$
i=1, \quad j=4, \quad k=3, \quad l=2,
$$


and those obtained from these by symmetry. For the specific choice (68), we can satisfy the hypothesis in the lemma by choosing $\hat{\mathbf{v}}_{1}=\left(1 / 2^{1 / 2}\right)\left(\hat{\mathbf{e}}_{2}+\hat{\mathbf{e}}_{3}\right)$ and $\hat{\mathbf{v}}_{2}=\hat{\mathbf{e}}_{1}$; we obtain the normal in the reference configuration to be:

$$
\hat{\mathbf{n}}_{i}=\frac{1}{2^{1 / 2}}\left(\hat{\mathbf{e}}_{2}+\hat{\mathbf{e}}_{3}\right), \quad \hat{\mathbf{n}}_{j}=\hat{\mathbf{e}}_{3}, \quad \hat{\mathbf{n}}_{k}=\frac{1}{2^{1 / 2}}\left(\hat{\mathbf{e}}_{2}+\hat{\mathbf{e}}_{3}\right), \quad \hat{\mathbf{n}}_{l}=\hat{\mathbf{e}}_{2} .
$$

The second solution corresponds to the choice

$$
i=1, \quad j=2, \quad k=5, \quad l=6,
$$

and those obtained from these by symmetry. For the specific choice (70), we can satisfy the hypothesis in the lemma by choosing $\hat{\mathbf{v}}_{1}=\hat{\mathbf{e}}_{2}$ and $\hat{\mathbf{v}}_{2}=\left(1 / 2^{1 / 2}\right)\left(\hat{\mathbf{e}}_{1}+\hat{\mathbf{e}}_{3}\right)$; we obtain the normals in the reference configuration to be

$$
\begin{aligned}
& \hat{\mathbf{n}}_{i}=\hat{\mathbf{e}}_{2}, \quad \hat{\mathbf{n}}_{j}=\frac{1}{\rho}\left[-\left(\eta_{1}^{0^{2}}+\eta_{2}^{\mathrm{o}^{2}}-2 \eta_{3}^{\mathrm{o}^{2}}\right) \hat{\mathbf{e}}_{1}+2\left(\eta_{1}^{\mathrm{o}^{2}}-\eta_{2}^{\mathrm{o}^{2}}\right) \hat{\mathbf{e}}_{2}+\left(\eta_{1}^{\mathrm{o}^{2}}+\eta_{2}^{\mathrm{o}^{2}}-2 \eta_{3}^{\mathrm{o}^{2}}\right) \hat{\mathbf{e}}_{3}\right], \\
& \hat{\mathbf{n}}_{k}=\hat{\mathbf{e}}_{2}, \quad \hat{\mathbf{n}}_{l}=\frac{1}{\rho^{\prime}}\left[\left(\eta_{1}^{\mathrm{o}^{2}}+\eta_{2}^{\mathrm{o}^{2}}-2 \eta_{3}^{\mathrm{o}^{2}}\right) \hat{\mathbf{e}}_{1}+2\left(\eta_{1}^{\mathrm{o}^{2}}-\eta_{2}^{\mathrm{o}^{2}}\right) \hat{\mathbf{e}}_{2}-\left(\eta_{1}^{\mathrm{o}^{2}}+\eta_{2}^{\mathrm{o}^{2}}-2 \eta_{3}^{\mathrm{o}^{2}}\right) \hat{\mathbf{e}}_{3}\right],
\end{aligned}
$$

where $\eta_{1}^{\mathrm{o}}=\frac{1}{2}\left(\alpha^{\mathrm{o}}+\delta^{\mathrm{o}}\right), \eta_{2}^{\mathrm{o}}=\frac{1}{2}\left(\alpha^{\mathrm{o}}-\delta^{\mathrm{o}}\right), \eta_{3}^{\mathrm{o}}=\beta^{\mathrm{o}}$ and $\alpha^{\mathrm{o}}, \delta^{\mathrm{o}}$ and $\beta^{\mathrm{o}}$ are lattice parameters of orthorhombic variants (20).

\subsection{Energy minimization}

We now show that the ferroelectric crystal can reduce its energy to zero by forming domain patterns. We also explain why energy minimization often forces a material to form very-fine-scale domain patterns.

Let us begin by considering an isolated ferroelectric single crystal and recalling the total energy $\hat{\mathcal{E}}$ in equation (23). Note that, for any $(\mathbf{F}, \mathbf{p}) \in \mathcal{W}$, the uniform state $(\mathbf{y}=\mathbf{F x}, \mathbf{p})$ makes the first term in the energy zero. However, the second term is not zero, except in very special circumstances. To see this, note that, if $\mathbf{n}^{\prime}$ is the normal to the body in the current configuration, it is not possible that $\mathbf{p} \cdot \mathbf{n}^{\prime}=0$ everywhere on the boundary unless of course the body is a plate in the current configuration. This in turn gives rise to electric fields since Maxwell's equation (2) has to be solved over all space. Thus, generically, the uniformly polarized state does not have zero energy.

Note, however, that, if $(\mathbf{F}, \mathbf{p}) \in \mathcal{W}$, then $(\mathbf{F},-\mathbf{p}) \in \mathcal{W}$. Further, according to lemma 1 we can form alternating layers of the state $(\mathbf{F}, \mathbf{p}),(\mathbf{F},-\mathbf{p})$ (with a suitable normal). Finally, if the layer thickness is small enough, we can satisfy $\mathbf{p} \cdot \mathbf{n}^{\prime}=0$ on average, and this allows us to reduce the second term of the energy to zero as well. This can be made precise using the arguments given by James and Kinderlehrer (1993). In particular for a sequence $\left(\mathbf{y}^{(k)}, \mathbf{p}^{(k)}\right)$ of alternating layers of thickness $1 / k$,

$$
\inf \hat{\mathcal{E}}=\lim _{k \rightarrow \infty}\left[\hat{\mathcal{E}}\left(\mathbf{y}^{(k)}, \mathbf{p}^{(k)}\right)\right]=0 .
$$

We could of course construct many other sequences which have zero energy in the limit. We can use banded domain patterns of the type in $\$ 3.3 .1$ which involve different deformation gradients and thus even satisfy suitable mechanical boundary conditions. This has been studied extensively in the context of martensitic phase transformations (Ball and James 1987, 1992, Bhattacharya 1991, 1992, Hane and Shield 1998). A general but simple characterization of all possible mechanical boundary conditions that can be achieved by sequences with zero energy is unfortunately unknown. 
Now consider a ferroelectric in contact with some conductor domains $C_{2}$ as shown in figure 1. Since we solve Maxwell's equation (2) with the condition $\nabla \phi=\mathbf{0}$ (see equation (3)) on the conductors (by allowing the formation of surface charges), it follows that no electric field is generated even if $\mathbf{p} \cdot \mathbf{n}^{\prime} \neq 0$ on that part of the ferroelectric that is in contact with the conductors. Therefore, we do not need fine patterns in the region that are 'shielded' by the conducting electrode.

\section{§4. Application to Design of Large-Strain Actuators}

\subsection{Flat-plate configuration}

Consider a ferroelectric crystal in the shape of a flat plate with electrodes on the two faces as shown in figure 10. Let $\Omega^{(h)}$ be the reference domain of the crystal:

$$
\Omega^{(h)}=\left\{\mathbf{x} \in \mathbb{R}^{3}:\left(x_{1}, x_{2}\right) \in S, 0<x_{3}<h\right\},
$$

where $S$ is the cross-section (bounded Lipschitz domain in two dimensions), $h$ is the thickness of the ferroelectric plate, and $\left\{x_{1}, x_{2}, x_{3}\right\}$ is relative to an orthonormal reference basis $\left\{\xi_{1}, \xi_{2}, \xi_{3}\right\}$. A uniaxial compressive stress and an electric field are applied to the crystal as also shown in figure 10 . We shall now show that, if the thickness of the crystal is much smaller than the lateral size and we apply a fixed electric field $\mathbf{e}_{0}$ and mechanical load derived from a constant tensor $\mathbf{T}_{0}$, that is $\mathbf{t}_{0}=\mathbf{T}_{0} \hat{\mathbf{n}}$ on $\partial \Omega$, then we can find the state of the crystal by minimizing the much simpler function

$$
G\left(\mathbf{F}, \mathbf{p} ; \mathbf{e}_{0}, \mathbf{T}_{0}\right)=W(\mathbf{F}, \mathbf{p})-\mathbf{e}_{0} \cdot \mathbf{p}(\operatorname{det} \mathbf{F})-\mathbf{T}_{0} \cdot \mathbf{F}
$$

instead of the functional $\mathcal{E}$ defined in equation (1). In particular, if the applied field is in the $\xi_{3}$ direction (i.e. $\mathbf{e}_{0}=e_{0} \xi_{3}$ ) and the applied stress is uniaxial compressive in the $\xi_{3}$ direction (i.e. $\mathbf{T}_{0}=-\sigma_{0} \xi_{3} \otimes \xi_{3}$ ) as shown in the figure, then

$$
G\left(\mathbf{F}, \mathbf{p} ; \mathbf{e}_{0}, \mathbf{T}_{0}\right)=W(\mathbf{F}, \mathbf{p})-e_{0}(\operatorname{det} \mathbf{F})\left(\mathbf{p} \cdot \xi_{3}\right)+\sigma_{0}\left(\xi_{3} \cdot \mathbf{F} \xi_{3}\right) .
$$

The idea behind replacing $\mathcal{E}$ by $G$ is the following. For the configuration under consideration, the electrostatic energy is negligible since the ferroelectric is shielded

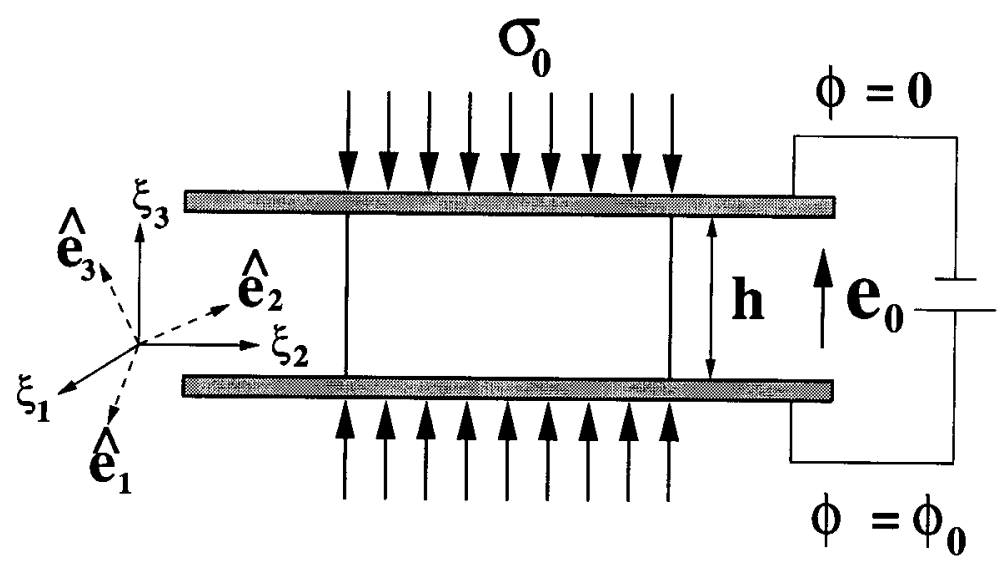

Figure 10. A ferroelectric crystal in the shape of a flat plate subject to a uniaxial compresive stress with magnitude $\sigma_{0}$ and electric field $\mathbf{e}_{0}$ obtained from a potential $\phi_{0}$. The physical and crystal bases are $\xi_{i}$ and $\hat{\mathbf{e}}_{i}$ respectively as shown. 
on the top and bottom faces, and the lateral surface is of negligible area. Furthermore, the minimizers are not forced to refine in the absence of the electric field, and therefore even the exchange energy is negligible. We are left with an integral over the body, which is minimized when the integrand (74) is minimized.

To make this precise, define

$$
\mathcal{E}^{(h)}(\mathbf{y}, \mathbf{p})=\frac{1}{\text { volume } \Omega^{(h)}} \mathcal{E}(\mathbf{y}, \mathbf{p})=\frac{1}{h|S|} \mathcal{E}(\mathbf{y}, \mathbf{p}),
$$

where $|S|$ denotes the area of $S$. We have the following theorem.

Theorem 1: Given constants $\mathbf{e}_{0}$ and $\mathbf{T}_{0}$,

$$
\lim _{h \rightarrow 0}\left[\min _{\mathbf{y}, \mathbf{p}}\left(\mathcal{E}^{(h)}(\mathbf{y}, \mathbf{p})\right)\right]=\min _{\mathbf{F}, \mathbf{p}}\left[G\left(\mathbf{F}, \mathbf{p} ; \mathbf{e}_{0}, \mathbf{T}_{0}\right)\right] .
$$

Proof: Since the first and last terms of the total energy $\mathcal{E}$ defined in equation (1) are non-negative, we easily find a lower bound:

$$
\begin{aligned}
\mathcal{E}^{(h)}(\mathbf{y}, \mathbf{p}) & \geqslant \frac{1}{h|S|} \int_{\Omega^{(h)}}\left[W\left(\nabla_{\mathbf{x}} \mathbf{y}, \mathbf{p}\right)-\mathbf{e}_{0} \cdot \mathbf{p}\left(\operatorname{det} \nabla_{\mathbf{x}} \mathbf{y}\right)-\mathbf{T}_{0} \cdot \nabla_{\mathbf{x}} \mathbf{y}\right] \mathrm{d} \mathbf{x} \\
& \geqslant \frac{1}{h|S|} \int_{\Omega^{(h)}}\left(\min _{\mathbf{F}, \mathbf{p}}\left[W(\mathbf{F}, \mathbf{p})-\mathbf{e}_{0} \cdot \mathbf{p}(\operatorname{det} \mathbf{F})-\mathbf{T}_{0} \cdot \mathbf{F}\right]\right) \mathrm{d} \mathbf{x} \\
& =\min _{\mathbf{F}, \mathbf{p}}\left[G\left(\mathbf{F}, \mathbf{p} ; \mathbf{e}_{0}, \mathbf{T}_{0}\right)\right] .
\end{aligned}
$$

It therefore suffices to show the upper bound

$$
\limsup _{h \rightarrow 0}\left(\min _{\mathbf{y}, \mathbf{p}}\left[\mathcal{E}^{(h)}(\mathbf{y}, \mathbf{p})\right]\right) \leqslant \min _{\mathbf{F}, \mathbf{p}}\left[G\left(\mathbf{F}, \mathbf{p} ; \mathbf{e}_{0}, \mathbf{T}_{0}\right)\right] .
$$

Given any constants $\mathbf{F}, \mathbf{p}$, define $\Omega_{\mathbf{F}}^{(h)}$ to be the image of $\Omega^{(h)}$ under $\mathbf{F}$ :

$$
\Omega_{\mathbf{F}}^{(h)}=\left\{\mathbf{y}: \mathbf{F}^{-1} \mathbf{y} \in \Omega^{(h)}\right\} .
$$

Note that, using equation (11), we have

$$
\mathcal{E}^{(h)}(\mathbf{F x}, \mathbf{p})=G\left(\mathbf{F}, \mathbf{p} ; \mathbf{e}_{0}, \mathbf{T}_{0}\right)+\mathcal{E}_{\text {elec }}^{(h)},
$$

where

$$
\mathcal{E}_{\text {elec }}^{(h)}=\frac{1}{h|S|} \int_{\Omega_{\mathrm{F}}^{(h)}} \mathbf{p} \cdot \nabla_{\mathbf{y}} \phi^{(h)} \mathrm{d} \mathbf{y}
$$

and $\phi^{(h)}$ solves Maxwell's equation (4) subject to $\phi^{(h)}=0$ on $S_{\mathbf{F}}^{(0)}$ and $S_{\mathbf{F}}^{(h)}$ which are the deformed top and bottom surfaces of the electrodes. We now change variables from $\mathbf{y}$ to $\mathbf{x}$ and define

$$
\tilde{\phi}^{(h)}(\mathbf{x})=\phi^{(h)}(\mathbf{F x}) \quad \text { and } \quad \nabla_{\mathbf{p}} \tilde{\phi}^{(h)}=\tilde{\phi}_{, 1}^{(h)} \xi_{1}+\tilde{\phi}_{, 2}^{(h)} \xi_{2}
$$


to obtain

$$
\begin{aligned}
\mathcal{E}_{\text {elec }}^{(h)} & =\frac{1}{h|S|} \int_{\Omega^{(h)}} \mathbf{p} \cdot\left(\mathbf{F}^{-\mathrm{T}} \nabla_{\mathbf{x}}\right) \tilde{\phi}^{(h)} \operatorname{det} \mathbf{F} \mathrm{d} \mathbf{x} \\
& =\frac{1}{h|S|} \int_{\Omega^{(h)}}\left(\mathbf{F}^{-1} \mathbf{p}\right) \cdot\left(\nabla_{\mathbf{p}} \tilde{\phi}^{(h)}+\tilde{\phi}_{, 3}^{(h)} \xi_{3}\right) \operatorname{det} \mathbf{F} \mathrm{d} \mathbf{x} \\
& =\frac{1}{h|S|} \int_{\Omega^{(h)}}\left(\mathbf{F}^{-1} \mathbf{p}\right) \cdot\left(\nabla_{\mathbf{p}} \tilde{\phi}^{(h)}\right) \operatorname{det} \mathbf{F} \mathrm{d} \mathbf{x}
\end{aligned}
$$

since $\tilde{\phi}^{(h)}=0$ on the top and bottom surfaces of the electrodes denoted by $S^{(0)}$ and $S^{(h)}$ in the reference configuration.

We now show that $\mathcal{E}_{\text {elec }}^{(h)}$ goes to zero with $h$. Recall from equation (12) that Maxwell's equation is equivalent to a variational principle. Therefore we can conclude by a change in variables that $\tilde{\phi}^{(h)}$ minimizes the functional

$$
\frac{1}{h|S|} \int_{\mathbb{R}^{3}}\left|\mathbf{F}^{-\mathrm{T}}\left(-\epsilon_{0} \nabla_{\mathbf{x}} \tilde{\phi}+\mathbf{F}^{\mathrm{T}} \mathbf{p}_{\Omega^{(h)}}\right)\right|^{2} \operatorname{det} \mathbf{F} \mathrm{d} \mathbf{x}
$$

over all $\tilde{\phi} \in H^{1}\left(\mathbb{R}^{3}\right)$ that vanishes on $S^{(0)}$ and $S^{(h)}$. To study this problem, it is convenient to introduce a second change in variables:

$$
z_{1}=x_{1}, \quad z_{2}=x_{2}, \quad z_{3}=\frac{1}{h} x_{3}
$$

and define

$\varphi(\mathbf{z}(\mathbf{x}))=\tilde{\phi}(\mathbf{x}), \Omega^{(1)}=\left\{\mathbf{z}:\left(z_{1}, z_{2}, h z_{3}\right) \in \Omega^{(h)}\right\}, S^{(1)}=\left\{\mathbf{z}:\left(z_{1}, z_{2}, h z_{3}\right) \in S^{(h)}\right\}$.

The variational problem (85) is the same as minimizing

$$
\mathcal{F}^{(h)}(\varphi)=\frac{1}{|S|} \int_{\mathbb{R}^{3}}\left|\mathbf{F}^{-\mathrm{T}}\left(-\epsilon_{0} \nabla_{\mathbf{p}} \varphi+\frac{1}{h} \varphi_{, 3} \xi_{3}+\mathbf{F}^{\mathrm{T}} \mathbf{p}_{\Omega_{\Omega^{(1)}}}\right)\right|^{2} \operatorname{det} \mathbf{F} \mathrm{d} \mathbf{z}
$$

over all $\varphi \in H^{1}\left(\mathbb{R}^{3}\right)$ that vanishes on $S^{(0)}$ and $S^{(1)}$. Let the minimizer be $\varphi^{(h)}$. Clearly,

$$
\mathcal{F}^{(h)}\left(\varphi^{(h)}\right) \leqslant \mathcal{F}^{(h)}(0)=|\mathbf{p}|^{2} \operatorname{det} \mathbf{F}
$$

Since $\left|\mathbf{F}^{-\mathrm{T}} \mathbf{v}\right|^{2} \geqslant \alpha|\mathbf{v}|^{2}$ for any vector $\mathbf{v}$ where $\alpha$ is the smallest eigenvalue of $\mathbf{F}^{-1} \mathbf{F}^{-\mathrm{T}}$, we conclude that

$$
\left\|\nabla_{\mathbf{p}} \varphi^{(h)}\right\|_{L^{2}} \leqslant C, \quad\left\|\frac{1}{h} \varphi_{, 3}^{(h)}\right\|_{L^{2}} \leqslant C
$$

for some constant $C$ independent of $h$. This also implies that

$$
\left\|\nabla_{\mathbf{z}} \varphi^{(h)}\right\|_{L^{2}} \leqslant C \text {. }
$$

Using the Poincaré inequality, we conclude that there exists a subsequence of $\varphi^{(h)}$ (not relabelled) and $\bar{\varphi} \in H^{1}\left(\mathbb{R}^{3}\right)$ that vanishes on $S^{(0)}$ and $S^{(1)}$ such that

$$
\varphi^{(h)} \rightarrow \bar{\varphi} \quad \text { in } H^{1}\left(\mathbb{R}^{3}\right) \text { as } h \rightarrow 0 .
$$

However, from the second of equations (90), we also have

$$
\varphi_{, 3}^{(h)} \rightarrow 0 \quad \text { in } L^{2}\left(\mathbb{R}^{3}\right) \text { as } h \rightarrow 0
$$


From the uniqueness of the weak limit, we conclude that

$$
\bar{\varphi}_{, 3}(\mathbf{z})=0 \quad \text { a.e. }
$$

Finally, since $\bar{\varphi}=0$ on $S^{(0)}$ and $S^{(1)}$ and since $\Omega^{(1)}$ is convex in $z_{3}$, we conclude that $\bar{\varphi}(\mathbf{z})=0$ on $\Omega^{(1)}$. Combining this with equation (92), we conclude that

$$
\frac{1}{|S|} \int_{\Omega^{(1)}}\left(\mathbf{F}^{-1} \mathbf{p}\right) \cdot\left(\nabla_{\mathbf{p}} \varphi^{(h)}\right) \operatorname{det} \mathbf{F} \mathrm{d} \mathbf{z} \rightarrow 0 \quad \text { as } h \rightarrow 0 .
$$

Changing variables back to $\mathbf{x}$ and comparing this with equation (84) we conclude that

$$
\mathcal{E}_{\text {elec }}^{(h)} \rightarrow 0 \quad \text { as } h \rightarrow 0 .
$$

Combining equations (81) and (96), we conclude that

$$
G\left(\mathbf{F}, \mathbf{p} ; \mathbf{e}_{0}, \mathbf{T}_{0}\right)=\limsup _{h \rightarrow 0}\left[\mathcal{E}^{(h)}(\mathbf{F x}, \mathbf{p})\right] \geqslant \underset{h \rightarrow 0}{\limsup }\left(\min _{\mathbf{y}, \mathbf{p}}\left[\mathcal{E}^{(h)}(\mathbf{y}, \mathbf{p})\right]\right) .
$$

This gives us the desired upper bound (79) and concludes the proof.

We conclude this section with the following remark. In the preceding, we assumed that the applied field $\mathbf{e}_{0}$ and load $\mathbf{T}_{0}$ are held fixed while the crystal deforms to its minimum-energy configuration. Experimentally, it is possible to apply a fixed mechanical load but it is very difficult to apply a fixed electrical field. Instead, experimentally it is convenient to apply a constant potential to the faces as shown in figure 10. Note now that the field changes as the crystal deforms, and $G$ in equation (74) should be replaced by

$$
\tilde{G}\left(\mathbf{F}, \mathbf{p} ; \phi_{0}, \sigma_{0}\right)=W(\mathbf{F}, \mathbf{p})-\frac{\phi_{0}}{h}(\operatorname{det} \mathbf{F})\left(\mathbf{p} \cdot \mathbf{F}^{-\mathrm{T}} \xi_{3}\right)+\sigma_{0}\left(\xi_{3} \cdot \mathbf{F} \xi_{3}\right),
$$

where we have applied a fixed electric potential $\phi_{0}$ and compressive load $\sigma_{0}$ as shown in figure 10. We can prove this using an argument very similar to that in theorem 1. Note that, if the deformation is small, then the difference between $\tilde{G}$ and $G$ in equation (75) with $e_{0}=\phi_{0} / h$ is negligible.

\subsection{Phase diagram}

The energy density $W$ has multiple wells as described in $\S 2$. The function $G$ (or $\tilde{G}$ ) in equation (74) (or equation (98)) inherits this multiwell structure; however, the heights of the individual wells are controlled by the applied loads $\sigma_{0}$ and $\phi_{0}$. In particular, $G$ does not satisfy the material symmetry condition (14), and thus the different variants do not necessarily have the same energy. In short, the applied fields and loads can bias one variant over others; similarly it can change the transformation temperatures. This information can be summarized in a ' $\sigma_{0}-\phi_{0}$ phase diagram' which shows the variant that minimizes $G$ for a given pair $\left(\sigma_{0}, \phi_{0}\right)$.

For example, consider a $\mathrm{BaTiO}_{3}$ single crystal at room temperature oriented such that the pseudocubic [001] axis is normal to the plate (i.e. the crystal axes $\left\{\hat{\mathbf{e}}_{i}\right\}$ coincides with the physical axes $\left\{\xi_{i}\right\}$ ). The phase diagram is shown schematically in figure 11. All tetragonal variants are equally preferred in the absence of applied field and load (at the point O). If we apply a moderate field and a small load as at (b), the crystal prefers the [001] polarized state; in contrast, if we apply a moderate load and a small field as at (a), the crystal prefers either a [100] or a [010] polarized state. 


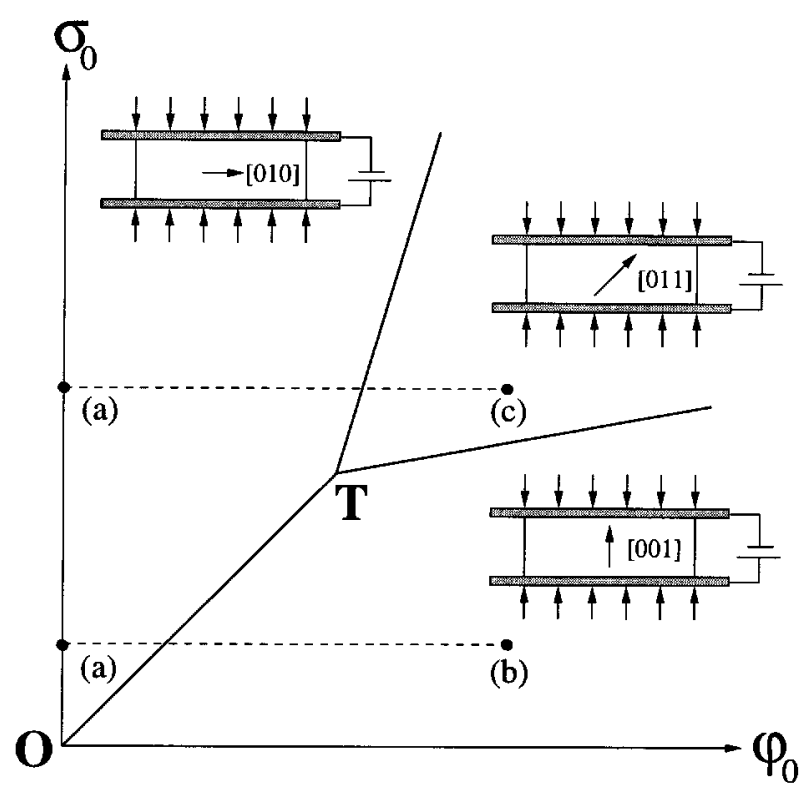

Figure 11. The field-stress phase diagram at room temperature for $\mathrm{BaTiO}_{3}$. The basic experimental idea is to apply a constant load and varying electric field to traverse the broken lines (a)-(b) or (a)-(c).

The exchange of stability takes place across the line OT. Finally, if we apply a very large field and load as at (c), the crystal transform s to the orthorhombic phase and is either [101] or [011] polarized. Note that $\mathrm{T}$ is the triple point where all the different states have equal energy. The details of this phase diagram and others for different temperatures and different crystal orientations have been presented elsewhere (Burcsu 2001).

\subsection{Maximum actuation strain}

The phase diagram in figure 11 and the result in lemma 1 suggest one way of obtaining large electrostriction. Suppose that we apply a fixed moderate load $\sigma_{0}$ and variable electric field $\phi_{0}$ so that we cycle along the broken line (a)-(b) in figure 11 . The material then cycles between the [010]- (or [100]-) and [001]-polarized states. Further, the domain wall described in $\S 3$ between these states provides a low-energy path between the two end states.

The phase diagram and the electrostriction depends on the orientation of the crystal. To find the orientation with the maximum electrostriction, let $\mathbf{R}$ be the rotation that maps the crystal basis to the physical basis:

$$
\hat{\mathbf{e}}_{i}=\mathbf{R} \xi_{i} .
$$

Then, the strain on switching from variant $i$ to variant $j$ is given by

$$
\max _{\mathbf{Q} \in \operatorname{SO}(3)}\left(\xi_{3} \cdot \mathbf{Q R} \mathbf{U}_{i} \mathbf{U}_{j}^{-1} \mathbf{R}^{\mathrm{T}} \xi_{3}\right)-1 ;
$$

so by maximizing over all crystal orientations and all pairs of variants, we conclude that the maximum actuation strain is given by 


$$
\epsilon_{\mathrm{A}}=\max _{\mathbf{R} \in \operatorname{SO}(3)} \max _{i, j} \max _{\mathbf{Q} \in \operatorname{SO}(3)}\left(\xi_{3} \cdot \mathbf{Q R} \mathbf{U}_{i} \mathbf{U}_{j}^{-1} \mathbf{R}^{\mathrm{T}} \xi_{3}\right)-1 .
$$

For tetragonal variants we find that the optimal rotations are $\mathbf{R}=\mathbf{Q}=\mathbf{I}$ and the optimal variants are $i=1$ and $j=3$, or $i=2$ and $j=3$. This is exactly the situation considered along the line (a)-(b) in figure 11. The maximum actuation strain is

$$
\epsilon_{\mathrm{A}}^{(\mathrm{t}-\mathrm{t})}=\frac{\eta_{2}^{\mathrm{t}}}{\eta_{1}^{\mathrm{t}}}-1
$$

which for $\mathrm{BaTiO}_{3}$ turns out to be

$$
\epsilon_{\mathrm{A}}^{(\mathrm{t}-\mathrm{t})} \approx 1.1 \%
$$

Therefore we can obtain an electrostriction of $1.1 \%$ with $\langle 001\rangle$-oriented $\mathrm{BaTiO}_{3}$ at room temperature by using an electromechanical loading path (a)-(b) in figure 11 . This is about ten times larger than the strain induced by the current PZT piezoelectric actuators. The corresponding value is about $6.3 \%$ in $\mathrm{PbTiO}_{3}$. Thus, a coupled electromechanical loading offers an attractive possibility for large electrostriction.

For the orthorhombic variants, the calculation of maximum actuation strain is more complicated than the above case. However, if the lattice parameters in equations (20) satisfy $\left|\alpha^{\mathrm{o}}-1\right| \ll 1,\left|\beta^{\mathrm{o}}-1\right| \ll 1$ and $\left|\delta^{\mathrm{o}}\right| \ll 1$, then

$$
\epsilon_{\mathrm{A}}^{(\mathrm{o}-\mathrm{o})} \approx \begin{cases}\left(\frac{\hat{\delta}^{2}+2}{2}\right)^{1 / 2}\left|\alpha^{\mathrm{o}}-\beta^{o}\right| & \text { if } \hat{\delta} \leqslant 2^{1 / 2}, \\ \hat{\delta}\left|\alpha^{\mathrm{o}}-\beta^{\mathrm{o}}\right| & \text { if } \hat{\delta} \geqslant 2^{1 / 2}\end{cases}
$$

where $\hat{\delta}=2 \delta^{\circ} /\left|\alpha^{\circ}-\beta^{\circ}\right|$. The optimal rotation $\mathbf{Q} \approx \mathbf{I}$, but now the optimal orientation $\mathbf{R}$ and variants depends on lattice parameters. If $\hat{\delta} \leqslant 2^{1 / 2}$, then the optimal pair can be chosen as $\left(\mathbf{U}_{1}^{\circ}, \mathbf{U}_{3}^{\text {o }}\right)$ with

$$
\mathbf{R}^{\mathrm{T}} \xi_{3} \approx \frac{\hat{\delta}}{\left[2\left(\hat{\delta}^{2}+2\right)\right]^{1 / 2}}\left(\xi_{1}+\frac{\hat{\delta}}{\left[2\left(\hat{\delta}^{2}+2\right)\right]^{1 / 2}-2} \xi_{2}-\frac{\hat{\delta}}{\left[2\left(\hat{\delta}^{2}+2\right)\right]^{1 / 2}+2} \xi_{3}\right) ;
$$

if $\hat{\delta} \geqslant 2^{1 / 2}$, then the optimal pair can be chosen as $\left(\mathbf{U}_{5}^{\circ}, \mathbf{U}_{6}^{o}\right)$ with

$$
\mathbf{R}^{\mathrm{T}} \xi_{3} \approx \frac{1}{2^{1 / 2}}\left(\xi_{2}+\xi_{3}\right)
$$

For $\mathrm{BaTiO}_{3}$ in the orthorhombic phase,

$$
\epsilon_{\mathrm{A}}^{(\mathrm{o}-\mathrm{o})} \approx 0.66 \% \text {. }
$$

We now consider the possibility of a phase transformation from the tetragonal to the orthorhombic phase. If the lattice parameters in equations (18) and (20) satisfy $\left|\eta_{1}^{\mathrm{t}}-1\right| \ll 1,\left|\eta_{2}^{\mathrm{t}}-1\right| \ll 1,\left|\alpha^{\mathrm{o}}-1\right| \ll 1,\left|\beta^{\mathrm{o}}-1\right| \ll 1$ and $\left|\delta^{\mathrm{o}}\right| \ll 1$, then

$$
\epsilon_{\mathrm{A}}^{(\mathrm{t}-\mathrm{o})} \approx \max \left\{\epsilon_{\mathrm{A}}^{(1)}, \epsilon_{\mathrm{A}}^{(2)}, \epsilon_{\mathrm{A}}^{(3)}, \epsilon_{\mathrm{A}}^{(4)}\right\},
$$


where

$$
\begin{array}{cc}
\epsilon_{\mathrm{A}}^{(1)}=\left|\eta_{1}^{\mathrm{t}}-\alpha^{\mathrm{o}} \pm \delta^{\mathrm{o}}\right|, & \epsilon_{\mathrm{A}}^{(2)}=\left|\eta_{2}^{\mathrm{t}}-\beta^{\mathrm{o}}\right| \\
\epsilon_{\mathrm{A}}^{(3)}=\left|\eta_{1}^{\mathrm{t}}-\alpha^{\mathrm{o}}+\frac{\eta_{2}^{\mathrm{t}}-\eta_{1}^{\mathrm{t}}}{2} \pm\left(\frac{\left(\eta_{2}^{\mathrm{t}}-\eta_{1}^{\mathrm{t}}\right)^{2}}{4}+\delta^{\mathrm{o}^{2}}\right)^{1 / 2}\right|, & \epsilon_{\mathrm{A}}^{(4)}=\left|\eta_{1}^{\mathrm{t}}-\beta^{\mathrm{o}}\right| .
\end{array}
$$

For $\mathrm{BaTiO}_{3}$

$$
\epsilon_{\mathrm{A}}^{(\mathrm{t}-\mathrm{o})}=\epsilon_{\mathrm{A}}^{(1)} \approx 1 \%
$$

and the associated optimal orientation $\mathbf{R}$ is

$$
\mathbf{R}^{\mathrm{T}} \xi_{3} \approx \frac{1}{2^{1 / 2}}\left(\xi_{2}+\xi_{3}\right), \text { for the pair }\left(\mathbf{U}_{1}^{\mathrm{t}}, \mathbf{U}_{5}^{\mathrm{o}}\right)
$$

Finally, we consider the possibility of a phase transformation from the tetragonal to the rhombohedral phase. If the lattice parameters in equations (18) and (22) satisfy $\left|\eta_{1}^{\mathrm{t}}-1\right| \ll 1,\left|\eta_{2}^{\mathrm{t}}-1\right| \ll 1,\left|\alpha^{\mathrm{r}}-1\right| \ll 1$ and $\left|\delta^{\mathrm{r}}\right| \ll 1$, then

$$
\epsilon_{\mathrm{A}}^{(\mathrm{t}-\mathrm{r})} \approx \max \left\{\epsilon_{\mathrm{A}}^{(1)}, \epsilon_{\mathrm{A}}^{(2)}\right\}
$$

where

$$
\begin{aligned}
& \epsilon_{\mathrm{A}}^{(1)} \approx \frac{1}{2}\left|\eta_{2}^{\mathrm{t}}+\eta_{1}^{\mathrm{t}}-\delta^{\mathrm{r}}-2 \alpha^{\mathrm{r}}+\left[\left(\eta_{2}^{\mathrm{t}}-\eta_{1}^{\mathrm{t}}\right)^{2}+2\left(\eta_{2}^{\mathrm{t}}-\eta_{1}^{\mathrm{t}}\right) \delta^{\mathrm{r}}+9 \delta^{\mathrm{r}^{2}}\right]^{1 / 2}\right| \\
& \epsilon_{\mathrm{A}}^{(2)} \approx\left|\eta_{1}^{\mathrm{t}}+\delta^{\mathrm{r}}-\alpha^{\mathrm{r}}\right| .
\end{aligned}
$$

The optimal pair can be chosen as $\left(\mathbf{U}_{1}^{\mathrm{t}}, \mathbf{U}_{1}^{\mathrm{r}}\right)$ with orientation close to

$$
\mathbf{R}^{\mathrm{T}} \xi_{3} \|\left\{\eta_{2}^{\mathrm{t}}-\eta_{1}^{\mathrm{t}}+\delta^{\mathrm{r}}+\left[\left(\eta_{2}^{\mathrm{t}}-\eta_{1}^{\mathrm{t}}\right)^{2}+2\left(\eta_{2}^{\mathrm{t}}-\eta_{1}^{\mathrm{t}}\right) \delta^{\mathrm{r}}+9 \delta^{\mathrm{r}^{2}}\right]^{1 / 2}\right\} \xi_{1}-2 \delta^{\mathrm{r}} \xi_{2}-2 \delta^{\mathrm{r}} \xi_{3}
$$

if $\epsilon_{\mathrm{A}}^{(\mathrm{t}-\mathrm{r})} \approx \epsilon_{\mathrm{A}}^{(1)}$, or

$$
\mathbf{R}^{\mathrm{T}} \xi_{3} \|\left(\xi_{2}-\xi_{3}\right)
$$

if $\epsilon_{\mathrm{A}}^{(\mathrm{t}-\mathrm{r})} \approx \epsilon_{\mathrm{A}}^{(2)}$

\subsection{Comparison with experiment}

Burcsu et al. (2000) have designed and built an experimental set-up to apply a constant mechanical load and variable electric field. They used a [001]-oriented single crystal (approximately $5 \mathrm{~mm} \times 5 \mathrm{~mm} \times 1 \mathrm{~mm}$ ) as suggested by the theoretical considerations above, applied a loading along the path (a)-(b) (see figure 11) and measured the strain. Their experimental result is shown in figure 12. Note that the strain is almost $0.9 \%$ for moderate fields of about $2 \mathrm{KV} \mathrm{mm}^{-1}$, and that this is close to the theoretically predicted value of $1.1 \%$. We do not know the reason for this small discrepancy at this moment but speculate the following. First, our theory finds absolute minimum while the material has to follow a suitable kinetic path which may become trapped in a local minimum. Second, the aspect ratio of the thickness to the lateral size of the specimen is $1: 5$ which may not be small enough to make the electrostatic end fields negligible. Further, there is some electric leakage on the electrodes in reality, giving rise to incomplete charge compensation. If the leakage is significant, the neglect of the depolarization energy is questionable. Finally, the specimen is not defect free; some microcracks extending from the edges to the centre of the specimen were observed prior to the experiment. This would cause electric 


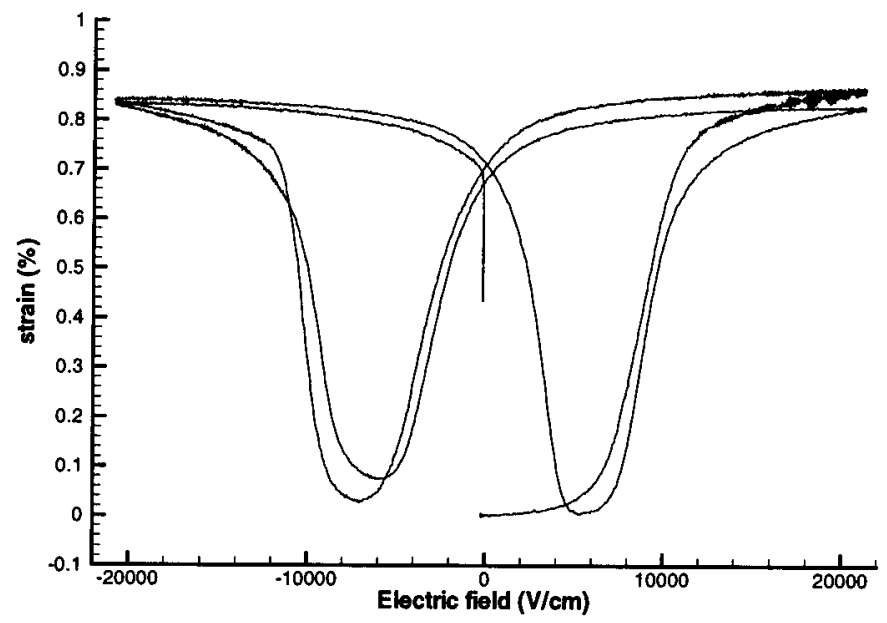

Figure 12. Experimental observation of actuation strain versus various electric voltage for $\mathrm{BaTiO}_{3}$. A constant uniaxial compressive stress with magnitude about $3.5 \mathrm{MPa}$ is applied on the specimen.

concentration around the crack tips which in turn could change the patterns of polarization switch. In any case, the agreement is encouraging and these specific issues will be addressed in future work.

\section{ACKNOWLEDGEMENTS}

We wish to thank R. D. James for numerous helpful and stimulating discussions. We are glad to acknowledge the partial support of the Air Force Office of Scientific Research (MURI: F49620-98-1-0433), the Army Research Office (DAAD19-99-1 0319) and the National Science Council of Taiwan (NSC 89-2212-E-002-103) .

\section{A P P E N D I X A}

Let $\hat{\mathbf{e}}_{1}=[100]_{c}, \hat{\mathbf{e}}_{2}=[010]_{c}$ and $\hat{\mathbf{e}}_{3}=[001]_{c}$ be a set of an orthonormal crystal basis relative to the pseudocubic axes. The point group $\mathcal{P}^{0}$ for a cubic lattice consists of 24 elements:

$$
\begin{gathered}
\mathbf{I}, \mathbf{R}^{\pi / 2}\left(\hat{\mathbf{e}}_{1}\right), \mathbf{R}^{\pi / 2}\left(\hat{\mathbf{e}}_{2}\right), \mathbf{R}^{\pi / 2}\left(\hat{\mathbf{e}}_{3}\right), \mathbf{R}^{\pi}\left(\hat{\mathbf{e}}_{1}\right), \mathbf{R}^{\pi}\left(\hat{\mathbf{e}}_{2}\right), \mathbf{R}^{\pi}\left(\hat{\mathbf{e}}_{3}\right), \mathbf{R}^{3 \pi / 2}\left(\hat{\mathbf{e}}_{1}\right), \mathbf{R}^{3 \pi / 2}\left(\hat{\mathbf{e}}_{2}\right), \\
\mathbf{R}^{3 \pi / 2}\left(\hat{\mathbf{e}}_{3}\right), \mathbf{R}^{\pi}\left(\frac{\hat{\mathbf{e}}_{1}+\hat{\mathbf{e}}_{2}}{2^{1 / 2}}\right), \mathbf{R}^{\pi}\left(\frac{\hat{\mathbf{e}}_{1}-\hat{\mathbf{e}}_{2}}{2^{1 / 2}}\right), \mathbf{R}^{\pi}\left(\frac{\hat{\mathbf{e}}_{2}+\hat{\mathbf{e}}_{3}}{2^{1 / 2}}\right), \mathbf{R}^{\pi}\left(\frac{\hat{\mathbf{e}}_{2}-\hat{\mathbf{e}}_{3}}{2^{1 / 2}}\right), \mathbf{R}^{\pi}\left(\frac{\hat{\mathbf{e}}_{3}+\hat{\mathbf{e}}_{1}}{2^{1 / 2}}\right), \\
\mathbf{R}^{\pi}\left(\frac{\hat{\mathbf{e}}_{3}-\hat{\mathbf{e}}_{1}}{2^{1 / 2}}\right), \mathbf{R}^{2 \pi / 3}\left(\frac{\hat{\mathbf{e}}_{1}+\hat{\mathbf{e}}_{2}+\hat{\mathbf{e}}_{3}}{3^{1 / 2}}\right), \mathbf{R}^{2 \pi / 3}\left(\frac{\hat{\mathbf{e}}_{1}+\hat{\mathbf{e}}_{2}-\hat{\mathbf{e}}_{3}}{3^{1 / 2}}\right), \mathbf{R}^{2 \pi / 3}\left(\frac{\hat{\mathbf{e}}_{1}-\hat{\mathbf{e}}_{2}+\hat{\mathbf{e}}_{3}}{3^{1 / 2}}\right), \\
\mathbf{R}^{2 \pi / 3}\left(\frac{\hat{\mathbf{e}}_{1}-\hat{\mathbf{e}}_{2}-\hat{\mathbf{e}}_{3}}{3^{1 / 2}}\right), \mathbf{R}^{4 \pi / 3}\left(\frac{\hat{\mathbf{e}}_{1}+\hat{\mathbf{e}}_{2}+\hat{\mathbf{e}}_{3}}{3^{1 / 2}}\right), \mathbf{R}^{4 \pi / 3}\left(\frac{\hat{\mathbf{e}}_{1}+\hat{\mathbf{e}}_{2}-\hat{\mathbf{e}}_{3}}{3^{1 / 2}}\right), \\
\mathbf{R}^{4 \pi / 3}\left(\frac{\hat{\mathbf{e}}_{1}-\hat{\mathbf{e}}_{2}+\hat{\mathbf{e}}_{3}}{3^{1 / 2}}\right), \mathbf{R}^{4 \pi / 3}\left(\frac{\hat{\mathbf{e}}_{1}-\hat{\mathbf{e}}_{2}-\hat{\mathbf{e}}_{3}}{3^{1 / 2}}\right)
\end{gathered}
$$


where

$$
\mathbf{R}^{\omega}(\hat{\mathbf{e}})=\cos \omega \mathbf{I}+(1-\cos \omega) \hat{\mathbf{e}} \otimes \hat{\mathbf{e}}+\sin \omega \hat{\mathbf{e}} \wedge
$$

is the proper rotation with $\hat{\mathbf{e}}$ as axis and $\omega$ as rotation angle.

Arlt, G., 1990, J. Mater. Sci., 25, 2655.

\section{REFERENCES}

Arlt, G., and Sasko, P., 1980, J. appl. Phys., 51, 4956.

Ball, J. M., and James, R. D., 1987, Arch.ration. Mech. Analysis, 100, 13; 1992, Phil. Trans. R. soc. A, 338, 389.

Bhattacharya, K., 1991, Acta metall. mater., 39, 2431; 1992, Arch.ration. Mech. Analysis, 120, 201; 1996, Contemporary Research in the Mechanics and Mathematics of Materials edited by R. C. Batra and M. F. Beatty (Barcelona: CIMNE), pp. 251-262.

Burcsu, E., 2001, PhD Thesis, California Institute of Technology.

Burcsu, E., Ravichandran, G., and Bhattacharya, K., 2000, Appl. Phys. Lett., 77, 1698.

Chou, C. C., and Wayman, C. M., 1997, J. Mater. Res., 12, 457.

Damuanovic, D., 1998, Rep. Prog. Phys., 61, 1267.

DeSimone, A., 1993, Archs. ration. Mech. Analysis, 125, 99.

DeSimone, A., and James, R. D., 1997, J. appl. Phys., 81, 5706; 2001, J. Mech. Phys. Solids, (in the press).

Devonshire, A. F., 1949, Phil. Mag., 40, 1040; 1951, ibid., 42, 1065; 1954, Phil. Mag. Suppl., 3,85 .

Ericksen, J. L., 1984, Phase Transformations and Material Instabilities in Solids, edited by M.

E. Gurtin (New York: Academic Press), pp. 61-78.

Fatuzzo, E., and Merz, W. J., 1967, Ferroelectricity (Amsterdam: North-Holland).

Goo, E. K. W., Mishra, R. K., and Thomas, G., 1981, J. appl. Phys., 52, 2940.

Gurtin, M., 1981. An Introduction to Continuum Mechanics (New York: Academic Press).

Hane, K. F., and Shield, T. W., 1998, Phil. Mag. A, 78, 1215.

Hooton, J. A., and Merz, W. J., 1955, Phys. Rev., 98, 409.

Hu, Y. H., Chan, H. M., Wen, Z. X., and Harmer, M. P., 1986, J. Am. Ceram. Seram. Soc., 69, 594.

Huang, X. R., Jiang, S. S., Xu, X. Y., Wang, J. Y., Guan, Q. C., Jiang, J. H., and Feng, D., 1995, Phys. Stat. sol. (a), 148, 611.

Huber, J. E., Fleck, N. A., and Ashby, M. F., 1997, Proc. R. Soc. (A), 453, 2185.

J AfFe, B., Cook, W. R., and JAfFe, H., 1971, Piezoelectric Ceramics (New York: Academic Press).

James, R. D., and Kinderlehrer, D., 1993, Phil. Mag. B, 68, 237.

James, R. D., and Wuttig, M., 1998, Phil. Mag. A, 77, 1273.

Jona, F., and Shirane, G., 1962, Ferroelectric Crystals (Oxford: Pergamon).

Li, Z., Chan, S. K., Grimsditch, M. H., and Zouboulis, E. S., 1991, J. appl. Phys., 70, 7327.

Li, Z., Foster, C. M., Dai, X. H., Xu, X. Z., Chan, S. K., and Lam, D. J., 1992, J. appl. Phys., 71, 4481.

Lines, M. E., and Glass, A. M., 1979, Principles and Applications of Ferroelectrics and Related Materials (Oxford University Press).

Little, E. A., 1955, Phys. Rev., 98, 978.

Mitsui, T., Abe, R., Furuhata, Y., Gesi, K., Ikeda, T., Kawabe, K., Makita, Y., Marutake, M., Nakamura, E., Nomura, S., Sawaguchi, E., Shiozaki, Y., Tatsuzaki, I., and Toyoda, K., 1969, Numerical Data and Functional Relationships in Sciences and Technology, edited by K. H. Hellwege (Berlin: Springer).

Park, B. M., and Chung, S. J., 1994, J. Am. Ceram. Soc., 77, 3193; 1996, Integrated Ferroelectrics, 12, 275; 1997, Phil. Mag. A, 75, 611.

Park, S. E., Wada, S., Cross, L. E., and Shrout, T. R., 1999, J. appl. Phys., 86, 2746.

Ricote, J., Whatmore, R. W., and Barber, D. J., 2000, J. Phys.: condens. Matter, 12, 323.

Shrout, T. R., and Park, S. E., 1997, J. appl. Phys., 82, 1804.

TanaKa, M., and Honjo, G., 1964, J. phys. soc. Japan, 19, 954.

UChino, K., 1988, Acta mater., 46, 3745.

Xu, Y., 1991, Ferroelectric Materials and Their Applications (Amsterdam: North-Holland). 